\title{
„Open book“-Frakturen des Mandibularbogens: Bilaterale Gelenkfortsatzfrakturen in Kombination mit Paramedian-/Medianfrakturen des Unterkiefers. Operative Behandlungsstrategien
}

\author{
Sebastian Schiel, Wenko Smolka, Christoph Leiggener, Gabriele Kaeppler, \\ Carl-Peter Cornelius
}

\section{Zusammenfassung}

In Analogie zu einem aufgeschlagenen Buch weit lateralwärts dislozierte Unterkieferhälften nach einem Gesichtsschädeltrauma sind Folge eines typischen Frakturmusters: die Symphysen-/Parasymphysen- bzw. Kinnregion ist aufgesprengt, gleichzeitig sind beide Kiefergelenkfortsätze nach Brüchen auf dem Niveau von Basis, Kollum oder Walze disloziert und/oder aus der Gelenkgrube luxiert. Aus derartigen Dreifachfrakturen resultieren massive Okklusionsstörungen (Kreuzbiss, frontal offener Biss), die nach Konsolidierung in Fehlstellung kaum funktionell kompensiert werden können. Daneben kann es zu einer ästhetisch ungünstigen Breitenzunahme des Untergesichts in Kombination mit einer Abnahme der hinteren Gesichtshöhe kommen. Aktuelle Konzepte tendieren daher $\mathrm{zu}$ einer operativen Versorgung sämtlicher Frakturzonen mit anatomisch korrekter Reposition und rigider Fixierung der Fragmente. Dieses aggressive Prozedere konnte sich erst auf der Grundlage von Fortschritten in der Behandlung von Kiefergelenkfortsatzfrakturen entwickeln, wo ein Paradigmenwechsel von der geschlossenen Behandlung zur Osteosynthese über offene transfaziale oder transorale Zugänge stattgefunden hat. Die Biomechanik, die klinische und bildgebende Diagnostik und die operative Gesamtstrategie der „Open book“Frakturen des Unterkiefers werden hier dargestellt. Die Differenzialindikation der operativen Zugänge, ebenso wie Entscheidungskriterien, wo (Gelenkfortsatzbereich oder Unterkiefermitte?) in der OP-Behandlungssequenz mit der Fixierung begonnen werden sollte sowie die Auslegung und Dimensionierung von Osteosynthesematerial finden dabei ausführliche Berücksichtigung.

"Open Book" Fractures of the Mandible: Bilateral Condylar Process Fractures Combined with Paramedial/ Medial Fractures of the Lower Jaw. Strategies for Surgical Treatment

Lateral displacement of the mandibular body divisions analogous to an open book after trauma to the facial skeleton are the result of a typical fracture pattern: a disjunction of the symphyseal/ parasymphyseal region goes along with bilateral condylar process fractures at the level of either the base of the process, the neck or the condylar head. The condylar process fragments are commonly displaced and/or dislo- cated from the glenoid fossa. Such triple mandibular fractures result in severe malocclusion (cross bite, frontal open bite) that can hardly be compensated for if the fractures consolidate in bony malalignment. Besides, the lower face may be widened and diminished in vertical height posteriorly in an aesthetically unpleasing manner. Present day concepts suggest open surgical treatment of all fracture sites with anatomic reduction and rigid fixation. This aggressive approach was only recently developed based on the advances in the treatment of condylar processes, where the paradigms have shifted from closed treatment to open reduction and osteosynthesis using a transfacial or a transoral access. The biomechanics, the clinical signs and symptoms, and imaging diagnostic work-up as well as the overall surgical strategy in the treatment of "open book" mandible fractures are presented in this article. The appropriate choice of the surgical access, the operative sequence at which site (condylar process or symphysis/ parsymphysis) to start with the fixation of fragments as well as the type, number, location and dimensions of osteosynthesis material are thoroughly outlined.

\section{Einleitung}

Frakturen in der Unterkiefermitte in Kombination mit dislozierten Frakturen beider Kiefergelenkfortsätze sind als besonders schweres Gesichtsschädeltrauma einzustufen, weil die Desintegration des Mandibularbogens zu einer weiter auseinander driftenden Translation und

OP-JOURNAL 2012; 28: 194-210

(c) Georg Thieme Verlag KG Stuttgart · New York DOI http://dx.doi.org/10.1055/s-0031-1298507 höhergradigen Rotation der Fragmente führen kann als die Dislokationen bei isoliert vorliegenden Frakturen in der Summe.

Nach der Aufsprengung des Mandibularbogens wird die beidseitige Aufweitung in der Transversaldimension nicht von noch intakten Kiefergelenkstrukturen limitiert (Abb.1). Stattdessen kann die Breitenzunahme im Untergesicht infolge der Medialkippung der Gelenkfortsätze soweit gehen, dass die lateral stehenden Fragmentenden die Seitenränder der Ge- lenkgruben bzw. die Jochbögen überragen. Durch die Höhenreduktion der aufsteigenden Unterkieferäste entsteht neben der Kreuzbiss- oder seitlichen Vorbeibiss-Situation gleichzeitig ein frontal offener Biss. Eine erfolgreiche Behandlung derartiger Unterkiefer-Dreifachfrakturen ohne Reposition und Fixation der Gelenkfortsätze sowie ohne eine stabile Wiederherstellung des Mandibularbogens in der Transversalen mit passgenauem Mittenschluss in der Symphyseal-/Parasymphysealregion ist schwer zu erreichen. Sogenannte „geschlossene“ 

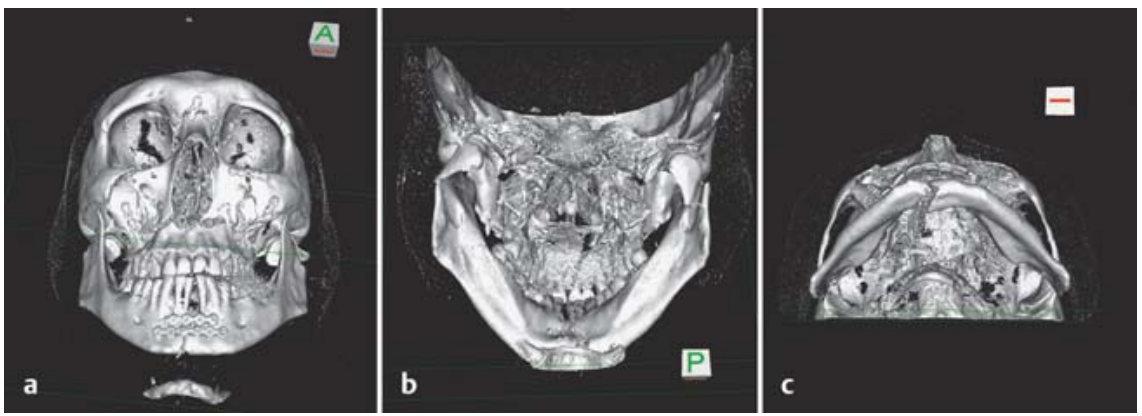

Abb. 1 a bis c „Open book“-Fraktur des Unterkiefers mit Osteosynthesen in Fehlstellung fixiert. Gleichzeitig haben Le-Fort-I- und -II-Frakturen sowie eine mediansagittale Gaumendachfraktur vorgelegen. Die Okklusion wurde in Referenz zum aufgebogenen Unterkiefer eingestellt, sodass kein Kreuzbiss vorhanden ist. a Ventralansicht: weit aufgebogene Untekieferhälften, Miniplatten über der Symphysenfraktur, wobei der Bruchspalt geschlossen erscheint, nach medial dislozierte gelenktragende Fragmente nach Gelenkhalsfrakturen beidseits. b Dorsalansicht: linguale Diastase in der Unterkiefersymphyse, Medialkippung der gelenktragenden Fragmente. c Kaudalansicht: Breitenzunahme durch abgeflachten Mandibularbogen.
Behandlungsverfahren, die insbesondere bei der Versorgung der Gelenkfortsatzfrakturen bis weit in die 1890er-Jahre dominierten, sind daher heute als Behandlungsoption nahezu verlassen. Aktuell stehen verschiedene OP-Techniken zur offenen Reposition und Osteosynthese zur Verfügung, deren Beschreibung und Indikation Gegenstand des folgenden Beitrags sind.

\section{Frakturmuster „Open book“}

Das Muster einer dislozierten Unterkiefer-Dreifachfraktur im Symphysen-/Parasymphysenbereich bzw. der Kinnregion und der Gelenkfortsätze beidseits gleicht einem aufgeschlagenen Buch (Abb. 1 und 2). Die Bezeichnung „Open book“Fraktur steht dabei plakativ für die um

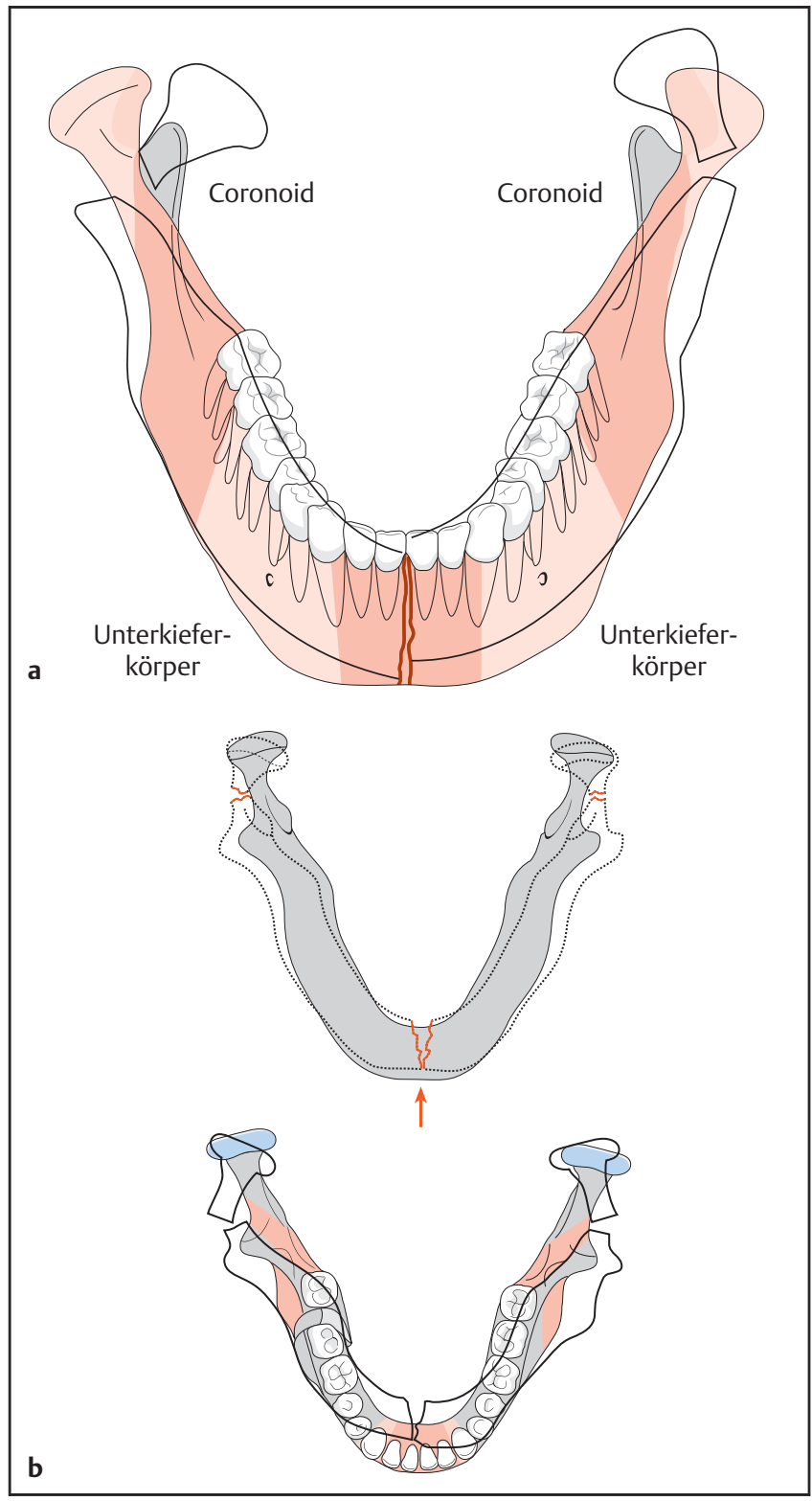

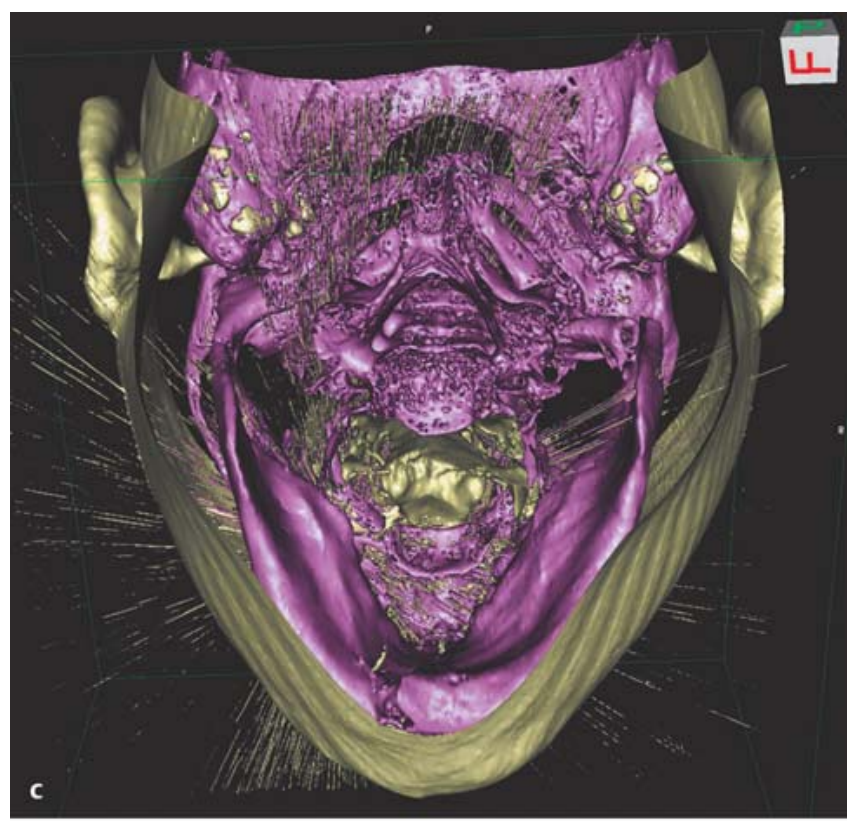

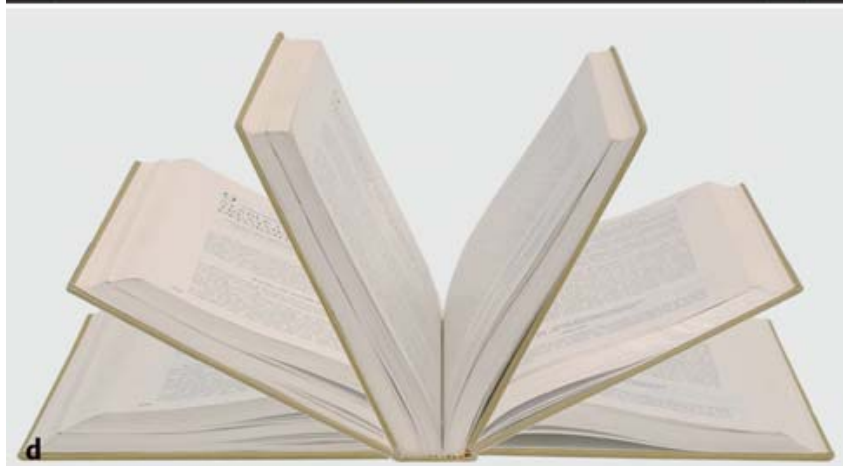

Abb. 2 a bis d „Open book“-Fraktur des Unterkiefers. a Schema von ventral: die Unterkieferhälften können umso weiter nach lateral aufklappen, je weiter kaudal die Gelenkfortsatzfrakturen lokalisiert sind. b Schema von kranial: beachte die Diastase auf der Lingualseite der Symphyse. c 3-D-CT von dorsal (Fallbeispiel) mit Darstellung des Hautmantels: rechts Medialkippung des Gelenkfortsatzes mit Luxation des Kopfes aus der Pfanne. d Offenes Buch als Metapher zur Beschreibung des Frakturmusters im Mandibularbogen. 


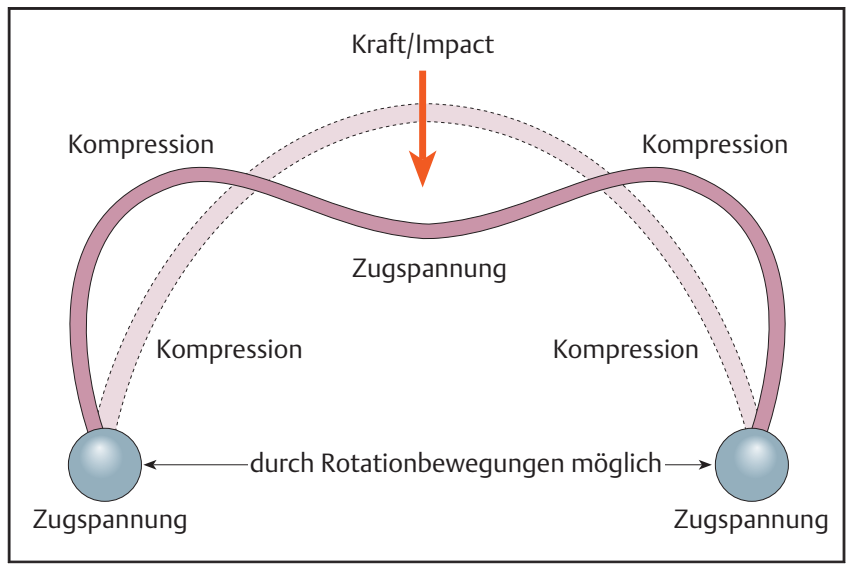

Abb. 3 Druck- und Zugzonen im Rundbogenmodell der Mandibula bei Krafteinwirkung von anterior. Die Gelenkfortsätze sind beweglich und deshalb als Kugeln dargestellt. Die Frakturentstehung mit Beginn auf der „Lingualseite“ der Symphyse und im Bereich beider Kiefergelenkfortsätze ist vorprogrammiert, da hier maximale Zugspannungen auftreten.

die Mitte lateralwärts aufrotierten Unterkieferhälften, die im Extremfall fast so flach wie Buchdeckel auf einer Tischplatte in der Frontalebene liegen können.

Die „Open book“-Fraktur des Unterkiefers ist auch als „Guardsman Fracture“ oder „Parade Ground Fracture“ bekannt, da Wachmänner und Paradesoldaten nach langem unbewegtem Stehen (orthostatischer Stress) in der Sonne mitunter vasovagale Synkopen erleiden und bei plötzlichem Verlust des Muskeltonus ohne Abfangmöglichkeit mit den oberen Gliedmaßen der Länge nach „ungebremst" auf das Kinn stürzen.

\section{Biomechanik}

Biomechanisch verhält sich der Unterkiefer bei Belastung in starker Vereinfachung wie ein freistehender Rundoder Halbkreisbogen mit frei drehbaren Enden, über dessen Gesamtlänge sich die Kräfte als Druckspannungen verteilen (Abb. 3).

In der Realität entspricht der Mandibularbogen aber nicht einer linearen Kurvenformation aus homogenem Knochenmaterial, sondern ist diskontinuierlich aufgebaut und hat Schwachstellen mit reduziertem Querschnitt und geringerer Knochendichte wie die Symphysen-/Parasymphysenregion, den Abschnitt mit den Foramina mentalia, die Kieferwinkel sowie die Kiefergelenkfortsätze mit den Kiefergelenkwalzen. An diesem „locus minores resistentiae“ konzentrieren sich Zugspannungen, aus denen - wie es auch von langen Röhrenknochen bekannt ist bevorzugt Frakturen resultieren [1-5]. In welcher Lokalisation Brüche am Unterkiefer auftreten, wird vom Vektor der einwirkenden Kraft bestimmt. „Open book“-Frakturen des Unterkiefers entstehen, wenn ein ausreichend energieKinnmitte gerichtet ist. In der Symphysen-/Parasymphysenregion kommt es infolgedessen zur direkten Fraktur. Die Außen- bzw. Bukkalfläche des anterioren Unterkieferkörpers wird dabei zunächst nur komprimiert, während die Zugkräfte die Innen- bzw. Lingualseite des Unterkiefers so stark überdehnen, dass der Knochen hier initial aufgesprengt wird und sich der Bruchspalt abrupt nach ventral ausbreitet [4].

Die Frakturen im Bereich der beiden Kiefergelenkfortsätze entstehen hingegen indirekt. Die Lateralflächen der aufsteigenden Unterkieferäste werden durch den Impuls nach außen konvex aufgebogen, bis die Zugspannung das Elastizitätsmodul des Knochens übersteigt und die Brüche herbeiführt. Die Kiefergelenkköpfe sind relativ frei beweglich in den Gelenkgruben gelagert. Neben der Rotationsbewegung werden die Gelenkköpfe auch axial (z.B. nach anteromedial) ausgelenkt, woraus Rupturen des Kapsel- und Bandapparats, Verlagerungen des Discus articularis, Luxationen und/oder Dislokationen in unterschiedlicher Richtung der gelenktragenden Fragmente resultieren können.

Immer wieder wurde die Vermutung geäußert, dass Zusammenhänge zwischen der Bezahnung im Molarengebiet und dem Typ, der Höhe und dem Ausmaß der Dislokation von Kiefergelenkfortsatzfrakturen bestehen, die sich jedoch bisher nicht bestätigen ließen [6]. Die Öffnungsstellung des Unterkiefers im Moment der Verletzung hat dagegen verifizierbaren Einfluss auf den Frakturtyp im Kiefergelenkfortsatzbereich $[7,8]$. In weit geöffneter Unterkieferposition werden die zur Fraktur führenden Kräfte bis in die Gelenkwalze oder den Gelenkhals weitergeleitet, in Schlussstellung ist da- reicher Impuls (Aufprall, Schlag) auf die gegen die Kiefergelenkfortsatzbasis betroffen [9].

\section{Klassifikation Gelenkfortsatzfrakturen}

Die bilateralen Gelenkfortsatzfrakturen bei einer „Open book“-Fraktur des Mandibularbogens können sich auf unterschiedlichem Niveau des Ramushinterrands abspielen mit Symmetrie oder Asymmetrie von Bruchhöhe und Dislokations- bzw. Luxationsgrad der gelenktragenden Fragmente. Die aktuelle AO-Klassifikation unterscheidet folgende Frakturen des Processus condylaris mandibulae:

- der Gelenkfortsatzbasis

- des Gelenkhalses („Kollum“)

- der Gelenkwalze (Synonyme: Gelenkkopf oder diakapituläre Frakturen)

Die Frakturspalten an der Gelenkfortsatzbasis und der Gelenkhalsregion (Kollum) sind in der Horizontalebene orientiert und verlaufen zumeist in abfallender Richtung von anterior-oben aus der Incisura sigmoidea nach dorsal-unten an den Ramushinterrand. Bei der „Open book"-Fraktur kommt es durch den Biegungsmechanismus an der Frakturstelle zur Achsabknickung (Abb. 4) nach medial, einer Seitwärtsverschiebung des kranialen Fragmentendes nach lateral oder medial („medial oder lateral override“) und einer longitudinalen Kontraktion. Der Gelenkkopf kann die Gelenkpfanne bei extremer Medialkippung des proximalen Fragments unter Zerreißung der Kapsel- und Diskusstrukturen in toto verlassen und somit zu einer echten Luxation führen. Der Zug des M. pterygoideus lateralis mit seinem Insertionsareal unterhalb des Gelenkkopfs kann eine nach vorne und medial gerichtete Komponente zur rein mechanisch verursachten Dislokation beitragen.

Im Unterschied zu den Basis- und Kollumfrakturen liegen diakapituläre oder Walzenfrakturen in der Sagittalebene [11-14], wobei der Bruchspalt schräg von lateral-oben nach medial-unten geneigt ist (Abb.5c). Funktionell relevant werden Walzenfrakturen, wenn sie den lateralen Pol einschließen und zur Höhenreduktion des Ramus führen. Das mediale Walzenfragment bleibt mit dem M. pterygoideus lateralis verbunden und kann durch den Muskelzug nach unten und vorne disloziert werden, wobei es aus der Gelenkgrube luxiert wird (Abb.5d). Ganz hohe horizontale Abbruchfrakturen werden ebenfalls den Walzenfrakturen zugerechnet. 


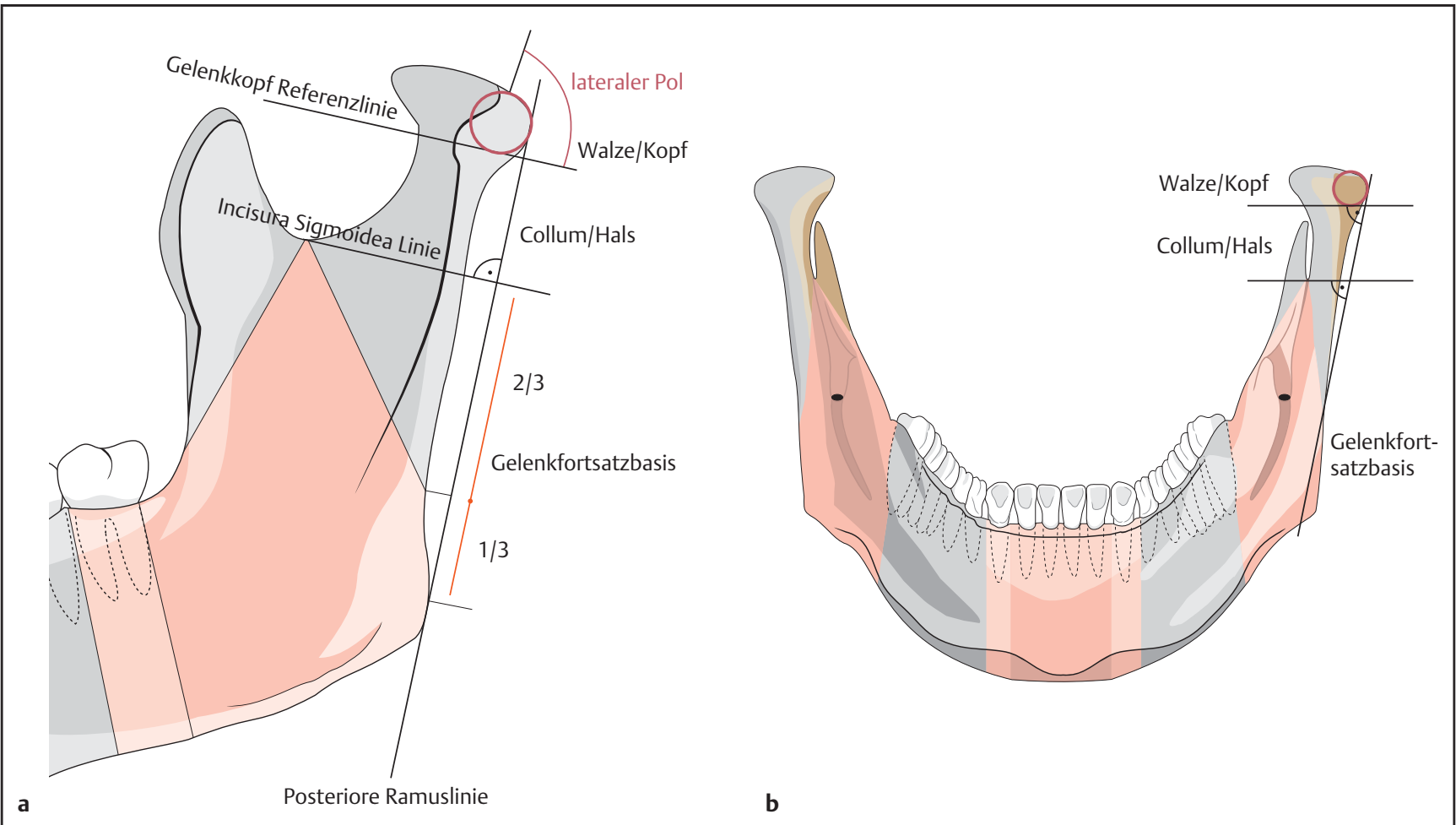

Abb.4a und b AO-Klassifikation der Kiefergelenkfortsatzfrakturen (in Anlehnung an Loukota et al. 2010 [10]). Zur Einteilung nach Höhe der Fraktur sind 2 Bezugslinien festgelegt, die senkrecht auf der posterioren Ramuslinie stehen: die „Incisura-sigmoidea-Linie“ trennt Gelenkfortsatzbasisfrakturen von Gelenkhals- bzw. Kollumfrakturen. Befindet sich die Fraktur zu mehr als $50 \%$ unterhalb dieser Linie, wird sie als Basisfraktur eingestuft, andernfalls als Kollumfraktur. Zur Differenzierung von Walzenfrakturen und Kollumfrakturen wird eine Tangente kaudal an einen Kreis gelegt, der den lateralen Pol der Gelenkwalze umgibt. Frakturen oberhalb dieser Referenzlinie werden als Walzenfrakturen klassifiziert, darunter lokalisierte sind wiederum den Kollumfrakturen zuzuordnen. a Lateralansicht des aufsteigenden Unterkieferasts. b Dorsalansicht des Mandibularbogens mit dem Hinterrand des aufsteigenden Unterkieferasts (Ramushinterrand).

\section{Klinische Symptomatik}

Die Leitsymptome der Unterkiefer„Open book“-Fraktur erklären sich aus dem Gesamtmuster des Dreifachbruchs. Die Dislokation der 4 Fragmente führt zu einer typischen Okklusionsstörung: frontal offener Biss durch Verlust der Ramushöhe und alleinigem Kontakt im Molarenbereich, Kreuzbiss oder seitlicher Vorbeibiss im Seitenzahngebiet durch Abflachung bzw. Verbreiterung des Mandibularbogens zusammen mit einer Rückwärtslage des abgeflacht deformierten Unterkiefers.

Ein erster äußerlicher Hinweis sind Prellmarken oder eine quere Riss-/Quetschwunde submental am Kinnunterrand. Im präaurikulären Wangen- bzw. Parotisbereich können deutlich sichtbare Auftreibungen vorhanden sein, die bei Palpation sehr schmerzhaft sind, da die Fragmentenden ins Weichgewebe ragen. Bei Luxationsfrakuren erscheint die Kiefergelenkgrube darüber leer. Oft sind Vorschub- und Seitwärtsbewegungen sowie die Kieferöffnung (i.S. einer Kieferklemme) eingeschränkt, da die Zugwir- kung der lateralen Pterygoidmuskulatur auf die Unterkieferspange unterbrochen ist. Die Rotations- und Vorwärtsbewegung des lateralen Pols der Kiefergelenkwalze ist infolge von Medialkippung oder anterokaudaler Dislokation des proximalen Fragments bei Öffnungsversuchen nicht mehr eindeutig durchzutasten. Wenn die Vorderwand des äußeren Gehörgangs einschließlich der Haut perforiert ist, blutet es aus dem Ohr.

Schon vorsichtiger Druck auf das Kinn intensiviert die Schmerzen im Gelenkbereich und an den Bruchstellen erheblich. Gleichzeitig wird dabei die Deformierbarkeit in der Mitte und an den Endausläufern des Mandibularbogens mit elastischer Rückstellung sicht- und palpierbar.

Bei Inspektion der Mundhöhle finden sich regelmäßig Hämatome im Mundvorhof und im anterioren Mundboden bzw. sublingual. Abhängig von der Größe der knöchernen Diastase und/oder einer Stufenbildung im Mittenbereich der Mandibula ist die befestigte Gingiva interdental und über dem Alveolarfortsatz aufgerissen und der Bruchspalt wird durch die klaffende Wunde direkt einsehbar. Falls diese Wunden sich in den anterioren Mundboden fortsetzen, treten nicht selten massive Blutungen auf, die durch eine rasche Reposition der Fragmente und zirkumdentale Drahtumschlingungen effektiv zu beherrschen sind.

\section{Bildgebende Diagnostik}

Eine „Open book“-Fraktur des Unterkiefers mit ihren Dislokationen lässt sich mit Einschränkungen in konventionellen Röntgenbildern darstellen. Als Minimalanforderung gilt die Diagnostik in wenigstens 2 - besser 3 - aufeinander senkrechten Ebenen. Zum Screening wird vielfach die Panoramaschichtaufnahme (PSA), oftmals auch als Orthopantomogramm (OPT) bezeichnet, eingesetzt. Die Symphysen-/Parasymphysenregion wird in der PSA durch Tangential- oder Summationsseffekte (z. B. Überprojektion von HWS oder Zungenbein) nur unklar wiedergegeben, Seitwärtsverschiebungen der proximalen Fragmente nach medial oder lateral sind nicht zu beurteilen. 


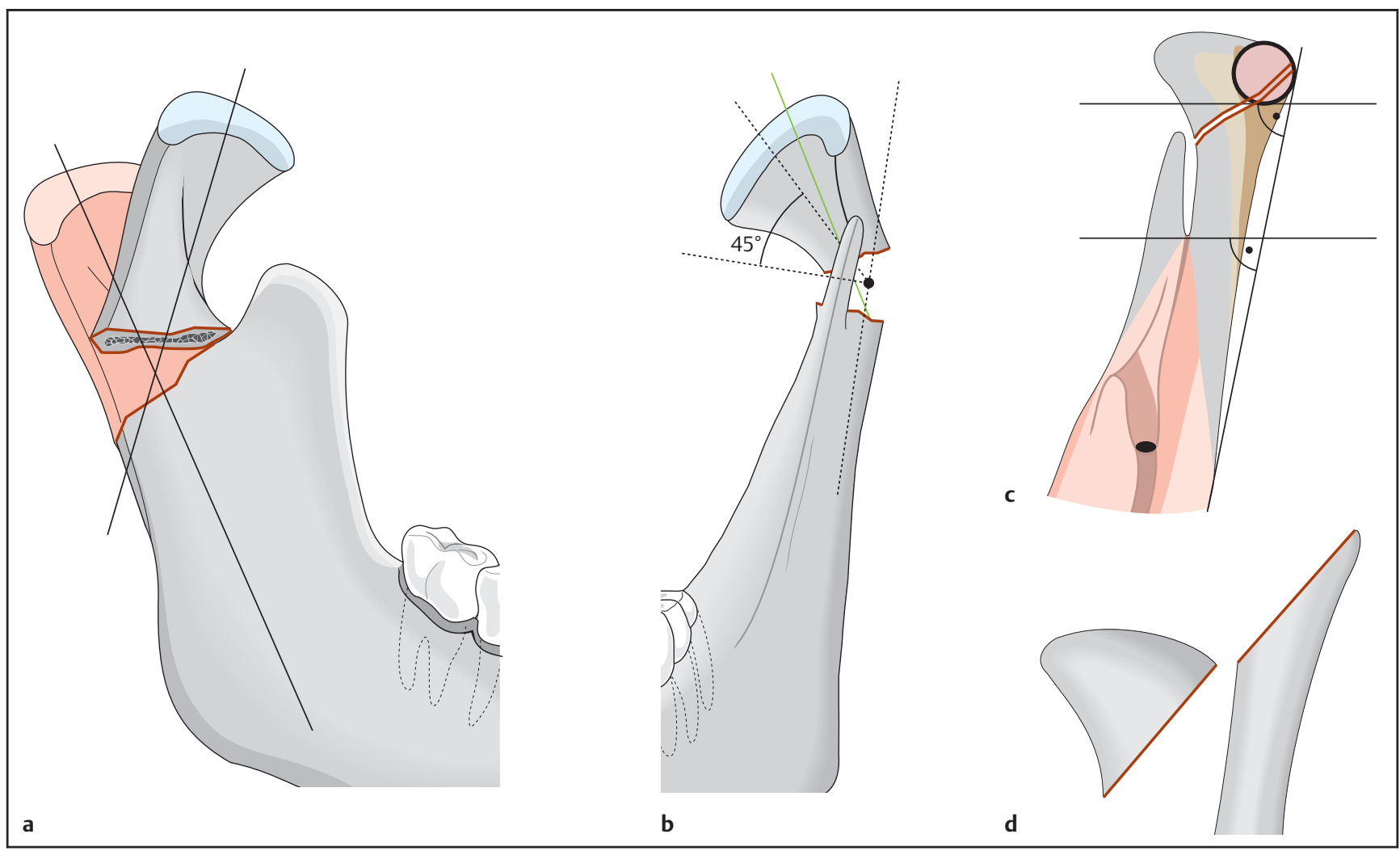

Abb. $\mathbf{5}$ a bis d Typische Dislokations- und Luxationsformen bei Frakturen des Processus condylaris mandibulae im Rahmen von „Open book“-Frakturen des Unterkiefers. a Gelenkfortsatzbasisfraktur rechts mit Dislocatio ad axim nach medial. b Kollumfraktur links mit $45^{\circ}$ Medialkippung des proximalen Fragments. c Nicht dislozierte Walzenfraktur links unter Mitbeteiligung des lateralen Gelenkpols rechts (Dorsalansicht). $\mathbf{d}$ Dislokation einer den lateralen Pol einbeziehenden Walzenfraktur nach unten und vorne.

Daneben werden zur der Beurteilung der Gelenkfortsätze die Unterkieferaufnahme im occipitofrontalen Strahlengang (Projektion nach Clementschitsch) und Schädelbasisaufnahmen zur Gelenkdarstellung (Parma und Schüller, Runström IV) angewandt. Die Aussagekraft dieser Aufnahmen verliert schnell an Präzision, wenn die Frakturen weit kranial, d.h. im Gelenkwalzenbereich, lokalisiert sind.

Mithilfe der Computertomografie oder der digitalen Volumentomografie wird eine detaillierte multiplanare (koronal, axial, sagittal) Beurteilung aller Frakturzonen und die 3-dimensionale Rekonstruktion des Mandibularbogens im Kontext zu den Fossae articulares, den Gehörgangswänden und den skeletalen Strukturen des Mittelgesichts, möglich. $\mathrm{Zu}$ den modernen digitalen Schnittbildgebungsverfahren gibt es daher strenggenommen keine Alternative mehr.

\section{Behandlungskonzepte}

Eine knöcherne Konsolidierung in originärer anatomischer Position der Fragmente, die Wiederherstellung einer ungestörten Gelenkfunktion und die
Reetablierung der prätraumatischen okklusalen Verhältnisse sind wichtige Erfolgskriterien der Behandlung von „Open book“-Frakturen.

Konservative Therapieansätze für alle Frakturstellen sind von vorneherein nicht miteinander vereinbar: zur Ausheilung der Fraktur in der Symphysen-/Parasymphysenregion wird nach geschlossener Reposition eine starre Ruhigstellung des Unterkiefers zwischen 4-6 Wochen Dauer notwendig. Die übliche starke Achsabweichung im Bereich der Kiefergelenkfortsatzbasis- oder Kollumfrakturen, gegebenenfalls sogar bei Kontaktverlust der Fragmentenden, verlangt hingegen eine Frühmobilisation nach einer vergleichsweise kurzen Immobilisationsphase von maximal 1-2 Wochen, damit es zur Ausbildung von Nearthrosen [11] oder auch Pseudarthrosen unter muskulärer Führung kommen kann. Eine Reposition bei einer Dislocatio ad axim ist konservativ nicht erreichbar und die Retention zur Konsolidierung in Fehlstellung kontraproduktiv. Eine Wiederabstützung des Ramus in der vertikalen Ausgangsdimension dürfte bei hoch lokalisierten Kollumfrakturen und Wal- zenfrakturen leichter zu erreichen sein, jedoch auch nur nach sofortiger Mobilisation. Die konservative Therapie von „Open book“-Frakturen des Unterkiefers durch Immobilisation und anschließende Funktionsübungen ist wenig erfolgversprechend und allenfalls in besonderen Konstellationen (nicht oder geringfügig dislozierte stabile Fragmente, OP-Kontraindikation aufgrund schwerwiegender Allgemeinerkrankungen, operative Therapie wird vom Patienten abgelehnt) zu rechtfertigen.

Als praktikables Konzept wird immer wieder eine Kombination von geschlossenen und offenen Therapieverfahren deklariert. Die Reposition und stabile Osteosynthese der ohne schwerwiegende Komplikationsmöglichkeiten angehbaren Fraktur in der Mitte des Mandibularbogens gilt als Grundvoraussetzung für den frühzeitigen Beginn zur funktionellen Behandlung der beiden Gelenkfortsatzfrakturen. Da sich bei beidseitigen Frakturen trotz langwieriger mandibulo-maxillärer Einstellung/Führung mit Gummizügen und intensiver Physiotherapie die Entwicklung eines frontoffenen Bisses regelhaft nicht verhindern lässt, 


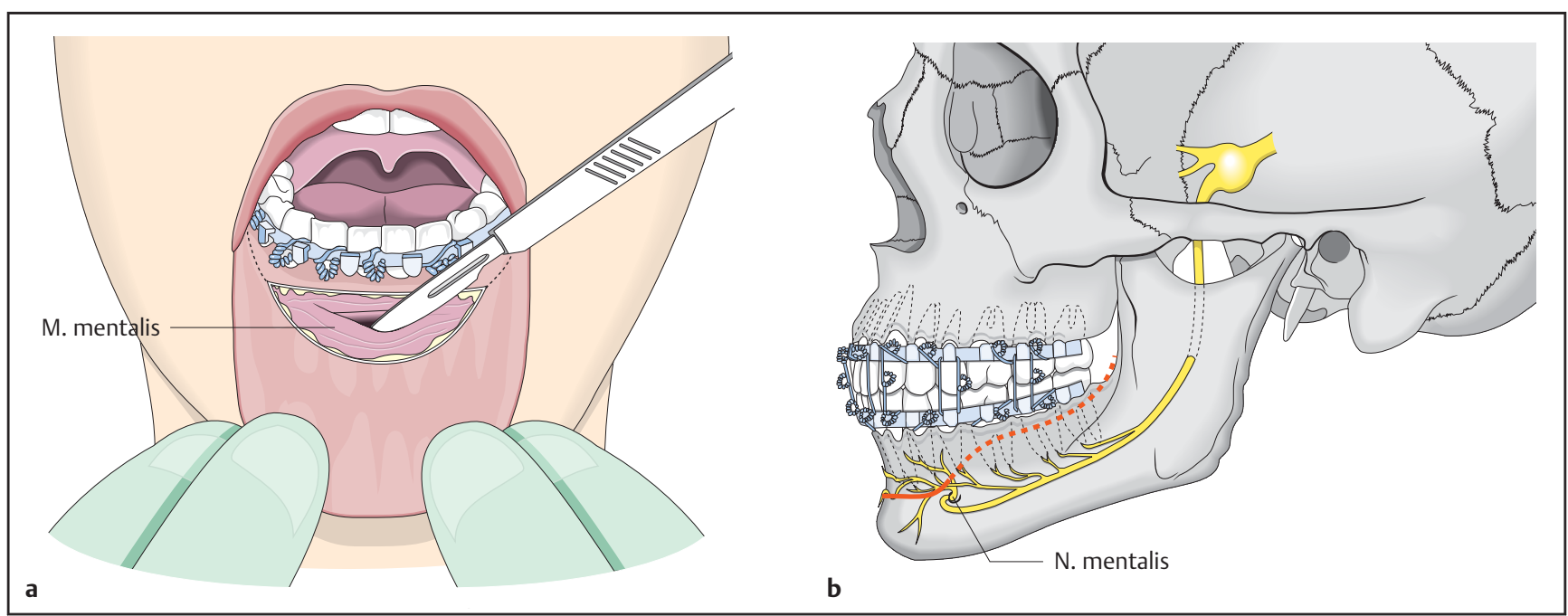

Abb. $\mathbf{6}$ a und b Wechselschnitt im anterioren Unterkiefervestibulum zur Darstellung der Symphysenregion. a Nach bogenförmiger Inzision wird ein Schleimhautläppchen in Richtung auf den Ansatz des M. mentalis gebildet. Der Muskel wird in Querrichtung scharf durchtrennt und das Kinn subperiostal freigelegt. b Verlauf der Inzisionslinie bei lateraler Erweiterung des vestibulären Zugangs zum Schutz des N. mentalis.

wird als präventive Strategie die Operation nur eines Gelenkfortsatzes empfohlen. Die am meisten gefürchtete Komplikation bei der operativen Versorgung der Gelenkfortsätze sind periphere Fazialisparesen beim Zugang oder durch Druck (Haken) oder Überdehnung, die durch das einseitige Vorgehen minimiert werden sollen. Ob zur unilateralen anatomischen Reposition und Fixation die mehr oder die minder schwer betroffene Seite ausgewählt wird, soll unter Berücksichtigung des potenziell risikoärmeren operativen Zugangswegs abgewogen werden. Da beide Kiefergelenke über den Mandibularbogen strukturell und funktionell in Verbindung stehen, bedeutet die einseitige Reparatur eine Kompromisslösung, nicht zuletzt weil bei Nearthrosenbildung in einem Gelenk, selbst bei anfänglich befriedigend adaptierten Funktionsabläufen und Wiedereinstellung der Okklusion auch im reparierten kontralateralen Gelenk längerfristig negative Auswirkungen (Arthrosis deformans) zu erwarten sind.

Angesichts der technischen Fortschritte in der Frakturversorgung von Kiefergelenkfortsatzfrakturen setzt sich die operative Versorgung aller 3 Brüche bei „Open book“-Frakturen des Unterkiefers mittlerweile allgemein durch.

Eine randomisierte prospektive Multicenterstudie konnte für Kiefergelenkfortsatzfrakturen auf allen Leveln die offene Reposition und interne Fixierung (ORIF) als die bessere Option gegenüber einer geschlossenen Immobilisationsbehandlung mit elastischer mandibulo- maxillärer Ruhigstellung belegen [15], wenn Achsabweichungen zwischen $10^{\circ}$ und $45^{\circ}$ und Verkürzungen der Ramushöhe $\geq 2 \mathrm{~mm}$ vorlagen. Die ORIF war insbesondere bei Patienten mit bilateralen Frakturen vorteilhaft. In einer retrospektiven Studie erwies sich ORIF im Vergleich zur geschlossenen Behandlung von bilateralen dislozierten Kiefergelenkfortsatzbasisfrakturen ebenfalls als überlegen [16].

\section{OP-Sequenz}

Zunächst sollten immer alle 3 Frakturzonen dargestellt werden, bevor irgendwo eine Reposition und Fixation vorgenommen wird. Im Schrifttum [17-19] wird die Reparatur der Unterkiefermitte als der essenzielle Schritt in der Behandlung von „Open book“-Frakturen betrachtet und deshalb dort mit der Wiederherstellung des Mandibularbogens begonnen.

Die Versorgung der Kiefergelenkfortsatzfrakturen an den Anfang der OP-Sequenz zu stellen, bietet bei starken Achsabweichungen, Luxationen der Walze und verkürzter Ramushöhe jedoch offensichtliche Vorteile. So sind die Ramus-Korpusfragmente einzeln mobiler und während der Reposition der Gelenkfortsätze leichter manipulierbar als eine bereits zusammengefügte Unterkieferspange. Bei Luxationsfrakturen lässt sich der Gelenkkopf in Verbindung mit einer ganzen Unterkieferhälfte zudem einfacher in die Fossa reponieren als am kleineren proximalen Fragment allein.
Darüber hinaus erleichtern die beiden wieder intakten Unterkieferhälften die Okklusionseinstellung. Durch die restituierte dorsokraniale Abstützung lässt sich die Bisslage auch bei Vorliegen einer Retro- oder Prognathie zuverlässig finden. Gleichzeitig wird die Gefahr einer Lateralrotation der Fragmente um die Sagittalachse vermindert. Diese Ausweichbewegung kann klinisch zunächst unbemerkt bleiben, da sich die damit einhergehende Disklusion auf die Innenseite der Okklusalflächen im Seitenzahngebiet beschränkt und sich einem Einblick entzieht. Schließlich reduziert die exakt reetablierte Okklusionsbeziehung die Stellungsvariablen beim Schluss der Bruchflächen in der Unterkiefermitte mit einem geringeren Risiko für eine Diastase auf der Lingualseite des Knochens.

\section{OP-Zugangswege}

\section{Symphysen-/Parasymphysenregion}

Zur operativen Versorgung im anterioren Unterkieferabschnitt wird in aller Regel ein transoraler Zugang verwendet. Im vorderen Vestibulum wird in ca. 10$15 \mathrm{~mm}$ Abstand vor der befestigten Gingiva von Eckzahn zu Eckzahn eine bogenförmige Schleimhautinzision angelegt und ein Mukosaläppchen in Richtung auf den Alveolarfortsatz bis an die Insertionsfläche des M. mentalis gebildet (Abb. 6a). Im Sinne eines Wechselschnitts wird der Muskel ca. $5 \mathrm{~mm}$ unterhalb seines Oberrands quer durchtrennt und die Kinnregion subperiostal freipräpariert. Die Muskelmanschette dient später einem 2-schichtigen Wundverschluss. 

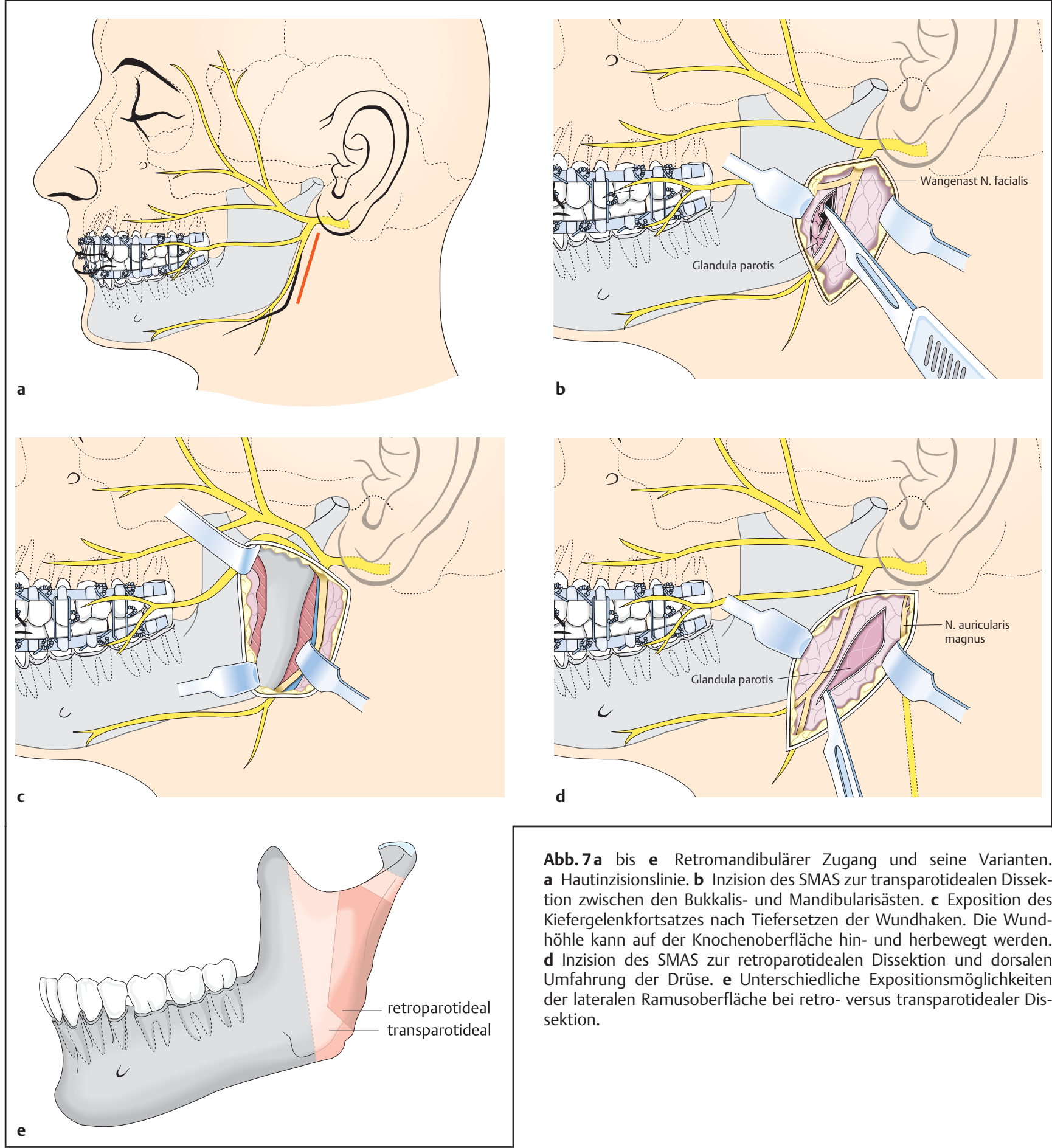

Abb.7a bis e Retromandibulärer Zugang und seine Varianten. a Hautinzisionslinie. b Inzision des SMAS zur transparotidealen Dissektion zwischen den Bukkalis- und Mandibularisästen. c Exposition des Kiefergelenkfortsatzes nach Tiefersetzen der Wundhaken. Die Wundhöhle kann auf der Knochenoberfläche hin- und herbewegt werden. d Inzision des SMAS zur retroparotidealen Dissektion und dorsalen Umfahrung der Drüse. e Unterschiedliche Expositionsmöglichkeiten der lateralen Ramusoberfläche bei retro- versus transparotidealer Dissektion.

Wird eine laterale Erweiterung des $\mathrm{Zu}-$ gangs dorsal der Eckzähne notwendig, dann muss zum Schutz des N. mentalis die Inzision in direkter Nähe zur Mukogingivalgrenze fortgesetzt werden (Abb. 6b).

Der vestibuläre Zugang gestattet keine oder nur eine unzureichende Kontrolle der Innenkortikalis, sodass man Gefahr läuft, nach der Fragmentreposition ver- bliebene linguale Diastasen zu übersehen. Zur Einsichtnahme kann eine bereits bestehende Riss-/Quetschwunde im Submentalbereich erweitert werden, eventuell ist man gezwungen, einen kleinen Hautschnitt vorzunehmen [20].

\section{Kiefergelenkfortsätze}

Die Zugänge zur offenen Reposition von Frakturen im Kiefergelenkfortsatzbereich richten sich nach der Höhenlokalisation des Bruchs und der Art der geplanten Osteosynthese:

- (prä-)aurikuläre oder auch retroaurikulär transmeatale (d.h. unter temporärer Durchtrennung des äußeren Gehörgangs) Zugangswege ermöglichen die Darstellung von sehr hoch gelegenen Kollumfrakturen und der sagittal verlaufenden Walzenfrakturen 


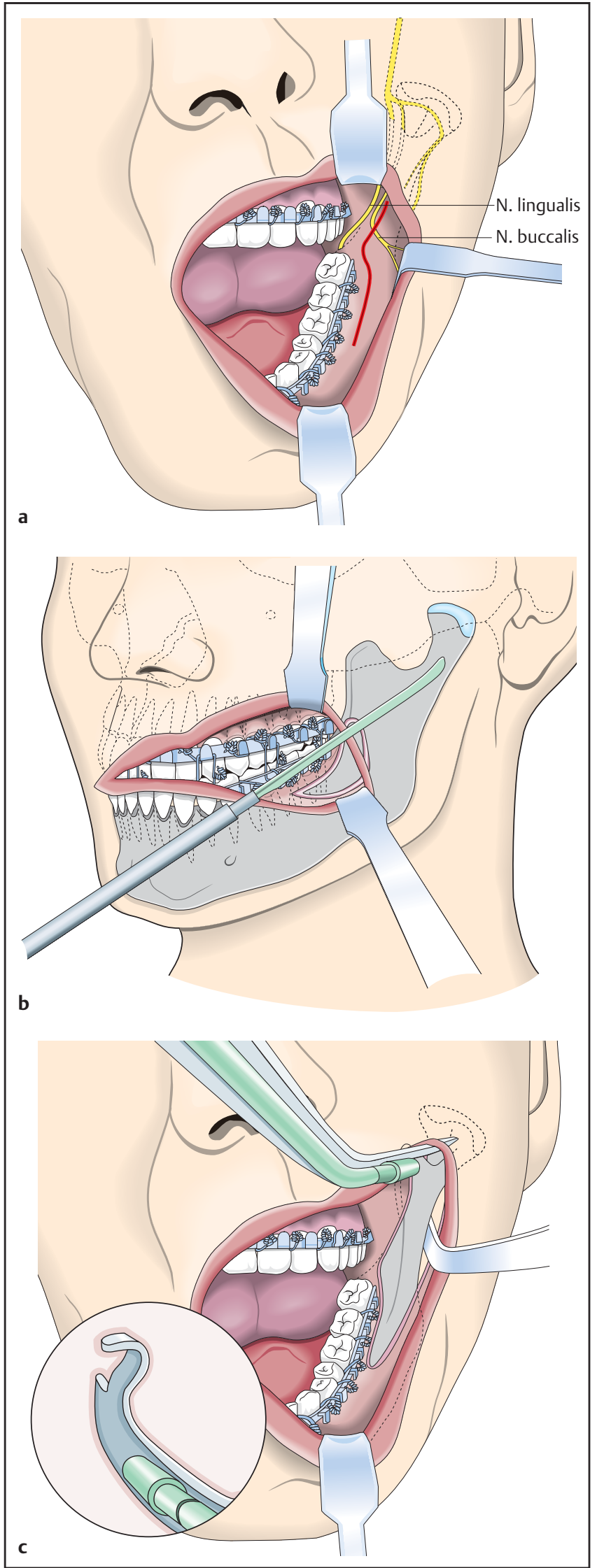

Abb. 8 a bis c

a Schleimhautinzisionslinie. b Exposition der Lateralfläche des aufsteigenden Unterkieferasts mit Kiefergelenkfortsatz nach Einsetzen der Wundhaken unter streng subperiostaler Dissektion. c Einsetzen der beleuchteten Wundhaken und Retraktoren zur Verbesserung der Übersicht nach Exposition des Kiefergelenkfortsatzbereichs.
- submandibuläre, perianguläre, retromandibuläre und transorale Zugänge eignen sich zur Versorgung von Kiefergelenkfortsatzbasis- und Kollumfrakturen

\section{(Prä-)aurikulärer Zugang}

Die Inzision kann in einer Hautfalte vor dem Tragus und dem vorderen Helixschenkel, auf der Tragus- und der Hinterkante des Crus helicis oder endaural transtragal erfolgen. Kranial kann der Schnitt in die behaarte Schläfe erweitert werden. Entlang des Ohrmuschelknorpels wird dann auf die äußere Lamina der Temporalisfaszie eingegangen. Die oberflächlichen Temporalgefäße, der N. auriculotemporalis und die Stirnäste des $\mathrm{N}$. facialis werden mit dem Hautweichgewebelappen nach anterior retrahiert, während das Gewebe vom Jochbogen nach ventral abgeschoben wird. Das äußere Faszienblatt wird auf Höhe der Fossa articularis über und oberhalb des Jochbogens in einer Länge von ca. $3 \mathrm{~cm}$ schräg nach vorne inzidiert. Auf der Oberfläche des temporalen Fettkörpers bzw. subperiostal lässt sich der Jochbogen dann nach ventral freipräparieren. Nach kaudal wird anschließend die Gelenkkapsel bis zum Übergang in den $\mathrm{Ge}$ lenkhals dargestellt. Die Freilegung von Kollumfrakturen erfolgt subperiostal ohne Eröffnung der Gelenkräume. Zur Darstellung der Lateral- und Dorsalfläche der Übergangs- und Gelenkkopfregion wird bei walzennahen und Walzenfrakturen eine T-förmige Inzision im unteren Gelenkspalt und in der Kapsel vorgenommen.

Der technisch aufwendige retroaurikuläre transmeatale Zugang soll eine bessere Übersicht auf die Dorsalfäche der Gelenkkopfregion erlauben und weniger häufig mit Fazialisläsionen einhergehen [21]. Nicht ganz unproblematisch ist hingegen die zwangsläufige sensible Denervation auf der gesamten Ohrmuschelrückseite und Präventivmaßnahmen zur Vermeidung von Gehörgangsstenosen.

Submandibulärer und periangulärer Zugang

Die traditionelle Inzision für den submandibulären Zugang verläuft in einer Hautfalte „2 Querfinger“ unterhalb des Kieferrands. Subkutis, Platysma und die obere Halsfaszie werden schichtweise durchtrennt, das faziale Gefäßbündel ligiert und nach kranial hochgeschlagen, um den lateral und kranial davon liegen- 


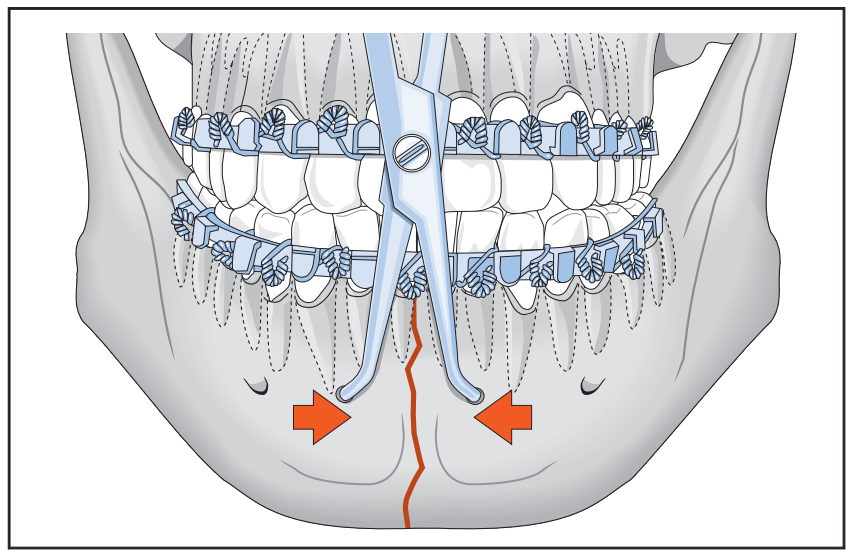

den Ramus marginalis mandibulae des $\mathrm{N}$. facialis aus dem Operationsfeld zu liften. Nach Freilegung und Inzision des Periosts über dem basalen Unterkieferrand wird dann der Masseteransatz scharf durchtrennt und der Ramus ascendens vom Kieferwinkel beginnend bis zur Fraktur im unteren und mittleren Gelenkfortsatzbereich freigelegt.

Der massetericomandibuläre Weichgewebemantel ist massiv und die Dissektionsstrecke bis zum Kiefergelenkfortsatz lang, weshalb die submandibuläre Schnittführung über $6-8 \mathrm{~cm}$ ausgedehnt sein muss. Wie der Name sagt, biegt die perianguläre Inzision in einer Länge von ca. $5 \mathrm{~cm}$ dorsal um den äußeren Kieferwinkel, liegt dabei nahe am Knochenrand und kann gedanklich als Fortführung des nach hinten ansteigenden submandibulären Schnitts gesehen werden. Die weitere Präparation wird über Wechselschnitte vorgenommen: zunächst wird das Platysma nach kranial $1,5-2 \mathrm{~cm}$ über den Unterkieferrand freipräpariert und im Faserverlauf nach dorsokranial eröffnet. Die in der Eigenfaszie des $M$. masseter liegenden bukkalen Fazialisäste werden darunter wie beim SMAS- (das Superficial Muscular Aponeurotic System geht in den Oberrand des Platysmas über) Face-Lift sichtbar. Zwischen der Verzweigung dieser Nervenäste wird der M. masseter entweder im Vertikalverlauf seiner Fasern [11] oder quer dazu und parallel zum Unterkieferrand [22] durchtrennt, um eine subperiostale Exposition des Ramus bis in die Kollumregion anzuschließen. Der perianguläre Zugang führt auf vergleichsweise kurzem Weg an die Kiefergelenkfortsatzbasis und in die Gelenkhalsregion, was das Handling und die Instrumentierung bei Reposition und Osteosynthese vereinfacht.
Abb. 9 Einbringen einer Repositionszange zur Kompression im Frakturbereich nach Reposition.

Retromandibulär-transparotidealer oder retroparotidealer Zugang

Der retromandibuläre Zugang in seinen beiden Dissektionsvarianten - transparotideal oder retroparotideal - hat sich nach unseren Erfahrungen zur einfachen und raschen Darstellung der unteren und mittleren Kiefergelenkfortsatzregion besonders bewährt.

Der vertikale Hautschnitt beginnt etwa $0,5 \mathrm{~cm}$ unterhalb des Ohrläppchens und erstreckt sich entlang des Ramus bis auf Höhe des Kieferwinkels (Abb.7a). Für die retroparotideale Dissektion ist vorgeschlagen worden, die Inzision bis zu $2 \mathrm{~cm}$ dorsal des Ramushinterrands $\mathrm{zu}$ platzieren, für die transparotideale Dissektion sollte der Schnitt weiter anterior auf der Ohrspeicheldrüse bzw. direkt über den Ramusstrukturen liegen.

Beim transparotidealen Vorgehen [23] wird das SMAS subkutan freipräpariert und vertikal eingeschnitten (Abb.7b), um auf die Parotiskapsel zu gelangen. Die Drüsenkapsel wird horizontal eröffnet und das Drüsenparenchym parallel zur Verlaufsrichtung der Fazialisäste stumpf zur Tiefe hin aufgespreizt. Die Dissektion bewegt sich dabei im Zwischenraum der Rr. buccales und mandibulares. Falls überhaupt Nervenäste sichtbar werden sollten, können sie mit dem Nervenstimulator identifiziert und abgedrängt werden. Sobald die Masseteroberfläche erreicht ist, wird die Muskelschicht im Faserverlauf durchtrennt und bis in die Frakturzone vom Knochen abgeschoben. Die Haken werden dann vom Hautrand aus tiefer gesetzt und retrahieren die Nerven im Schutzmantel des Drüsenparenchyms (Abb. 7c).

Bei der retroparotidealen Dissektion wird die Ohrspeicheldrüse intakt gelassen und von der Rückseite aus ange- hoben. Von der posterior lokalisierten Hautinzision aus wird zuerst das SMAS freigelegt und über den dorsalen Ausläufern der Parotis schräg inzidiert. Im nächsten Schritt wird die Kapsel der Parotis freigelegt und nach hinten stumpf umfahren bis der Masseter erreicht ist. Die Drüse wird nach ventral etwas von der Muskeloberfläche abgehoben, um den Muskel entlang des Ramushinterrands zu durchtrennen und dann vom Gelenkfortsatz abzulösen. Der Passageweg bei der retroparotidealen Dissektion verläuft zwischen den Ästen des N. auricularis magnus am Vorderrand des M. sternocleidomastoideus und den Mandibularisästen des N. facialis, die von der Drüse umgeben sind (Abb. 7d)

Mit einem transparotidealen Prozedere ist im Vergleich zur retroparotidealen Vorgehensweise eine umfangreichere Exposition der Ramusregion möglich (Abb. 7d), weil die Distanz zur Knochenoberfläche kürzer ist und die abgelösten Gewebeschichten besser über dem Knochen hin- und herbewegt werden können.

Transoraler (endoskopisch unterstützter) Zugang

Der transorale Zugang wurde erstmals von Silverman [24] als Zugangsweg zum Kiefergelenkfortsatz beschrieben und vermeidet das Risiko einer Schädigung des N. facialis sowie eine äußerlich sichtbare Narbe. Allerdings muss dabei anfänglich eine längere Operationszeit mit einem unübersichtlicheren OP-Situs in Kauf genommen werden. Seit seiner Renaissance durch die endoskopisch unterstützte Modifikation [25] hat er sich als alternativer operativer Zugangsweg bei Kiefergelenkfortsatzfrakturen durchgesetzt und bei Vorhandensein eines Spezialinstrumentariums $\left(90^{\circ}\right.$ gewinkelter Bohrer/Schraubendreher, Lichthaken) und hinreichender Erfahrung des OPTeams bewährt [26-28].

Die typische Schnittführung beim transoralen Zugang erfolgt entlang der Vorderkante des aufsteigenden Unterkieferasts über die Linea obliqua mit Fortsetzung ins laterale Unterkiefervestibulum entlang der mukogingivalen Grenze bis auf Höhe des 1. Prämolaren. Nach Inzision der Mukosa unter Schonung des N. buccalis (Abb. 8a) und nachfolgender scharfer Periostdurchtrennung erfolgt unter streng subperiostaler Abpräparation die Darstellung der Lateralfläche des aufsteigenden Unterkieferasts aus der 


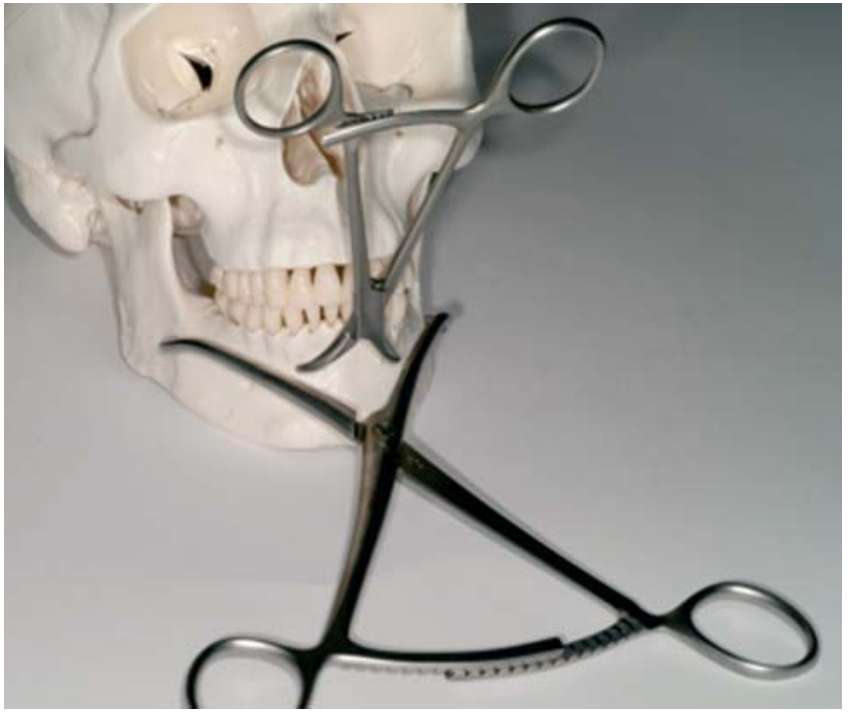

Abb. 10 Einsetzen einer weiteren Repositionszange im Kieferwinkelbereich zur Verhinderung eines lingualen Spalts.

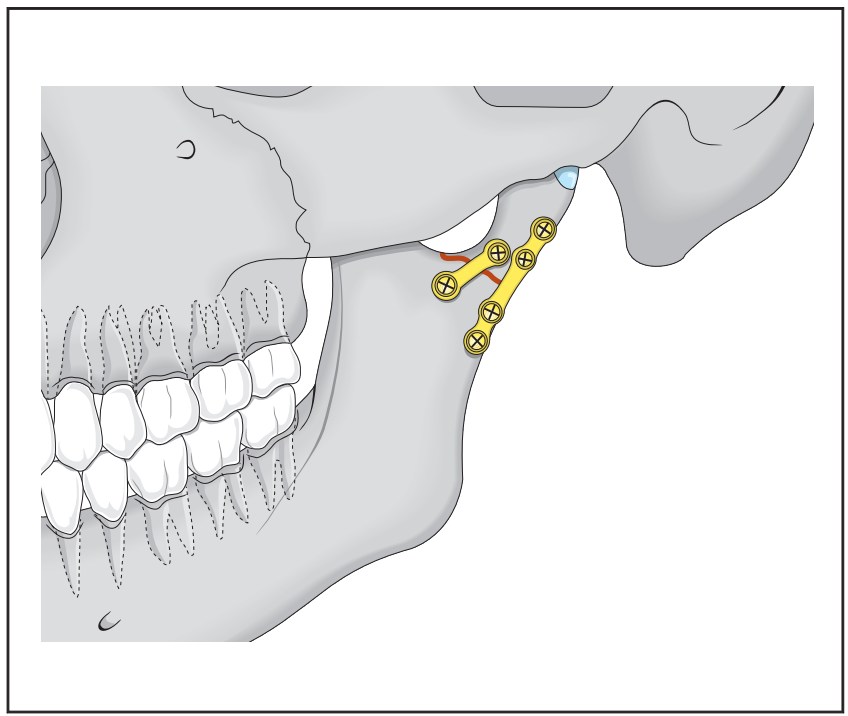

Abb. 11 Positionierung von 2 Miniosteosyntheseplatten am Vorderund Hinterrand des Kiefergelenkfortsatzes.

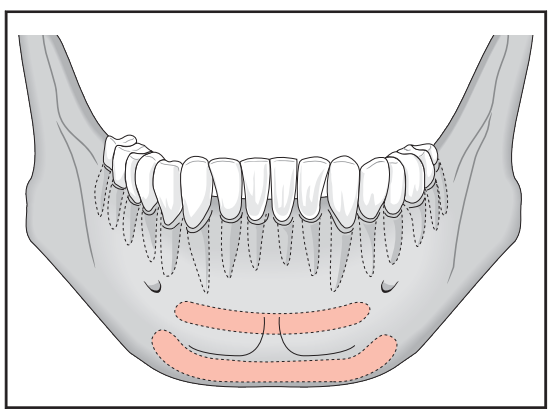

Abb. 12 Optimale Position des Osteosynthesematerials (Zugschrauben bzw. Osteosyntheseplatten) in der Unterkiefermitte entsprechend den Kraftlinien.

Kieferwinkelregion bis in den Bereich der Incisura semilunaris (Abb. 8b). Dabei müssen inferiore Anteile des Ansatzes des M. temporalis vom Muskelfortsatz des Unterkiefers abgelöst werden, um eine ausreichende Übersicht zu erreichen. Bei streng subperiostaler Präparation werden Verletzungen des M. masseter vermieden und eine Übersicht ohne Blutung bleibt erhalten. Das Einbringen von beleuchteten Wundhaken mit Lichtleitern und speziellen Retraktoren führt zu einer deutlichen Verbesserung der Übersicht (Abb. 8c). Nach Freilegung des Hinterrands des aufsteigenden Unterkieferasts und der Kiefergelenkfortsatzregion erfolgt die vollständige Darstellung in Bereichen limitierter Exposition durch Verwendung eines Endoskops (30 $\mathrm{Op}$ tik) in Verbindung mit einer Xenonlichtquelle.

\section{Mandibulo-maxilläre Fixation}

Die mandibulo-maxilläre Fixation (MMF) ist eine starre oder elastische Verbindung des Unterkiefers mit dem Oberkiefer in einer für die Okklusion korrekten Position. Da unabhängig von der Wahl des jeweiligen Therapiekonzepts (nicht operativ/operativ) Maßnahmen zur Sicherung der Okklusion ergriffen werden müssen, sollen die beiden wichtigsten Techniken der mandibulo-maxillären Fixation (MMF) kurz vorgestellt werden. Die Kieferbruchschiene, die noch bis Anfang der Jahrtausendwende als Goldstandard der Okklusionssicherung angesehen wurde [29], wird zunehmend durch die MMF-Schrauben abgelöst.

\section{Drahtschienenverbände (sog. Kieferbruchschienen)}

Es stehen eine Vielzahl von Drahtschienenverbänden, von individuell angefertigten bis hin zu präfabrizierten, zur Verfügung. Dabei scheinen die DrahtbogenKunststoffschienen nach Schuchardt, die Drahtschienung nach Obwegeser, die Sauer-Schiene und die Kappenschienen im deutschsprachigen Raum am weitesten verbreitet zu sein. Allen Drahtschienenverbänden gemein ist, dass ein Draht oder Drähte intraoperativ den Zahnreihen angebogen und unterhalb des Zahnäquators an den Zähnen mit Draht fixiert wird. Dies erklärt auch die ungünstige Auswirkung auf das Parodontium. Über die an den Drahtschienenverbänden bestehenden Häkchen oder Ösen wird die MMF über straffe Gummis oder Draht vorgenommen.

\section{MMF-(IMF-)Schrauben}

Aufgrund der technisch einfachen, zeitsparenden Handhabung und der geringen Verletzungsgefahr für den Operateur finden MMF-Schrauben zunehmend Verwendung. Die Methode der Fixation über MMF-Schrauben wurde erstmals von Arthur und Berardo beschrieben [30]. Eine Vielzahl von unterschiedlichen MMFSchraubentypen ist im Handel erhältlich. Die selbstbohrende MMF-Schraube wird zwischen die Zahnwurzel zweier benachbarter Zähne eingebracht, wobei die Position nach Frakturlokalisation und Wurzelabstand ausgewählt wird. Zur temporären intraoperativen MMF bei einer operativen Frakturversorgung reicht in der Regel ein sog. 4er-Muster, bei dem jeweils 1 MMF-Schraube in der prämolaren Region jedes Kieferquadranten verwendet wird. Die Gefahr liegt in der Zahnwurzelverletzung, wobei periphere Wurzelverletzungen wahrscheinlich folgenlos abheilen. Im Allgemeinen erfolgt nach der Vorbereitung der jeweiligen MMF-Technik (Einligieren von Kieferbruchschienen oder Insertion von MMFSchrauben) die Darstellung der Frakturen zur anschließenden offenen Reposition und Osteosynthese unter MMF.

\section{Reposition}

Die Herstellung der ursprünglichen Anatomie ist Ziel einer jeglichen Reposition der Fragmente und die beste Voraussetzung zur Wiederherstellung der Funktion. Eine offene exakte Reposition kann fast immer erreicht werden und ist bei nahezu allen Frakturtypen möglich. 


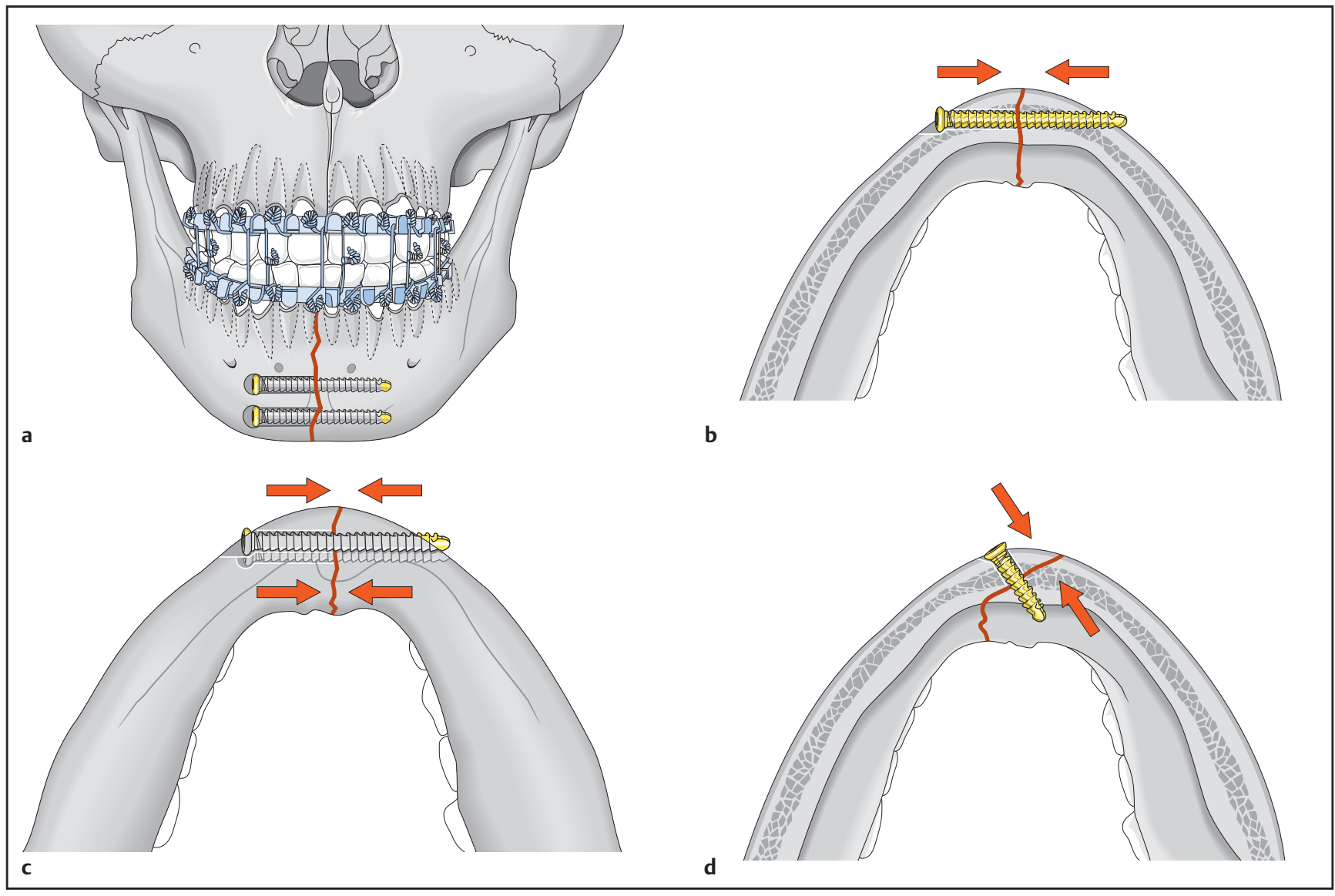

Abb. 13a bis d a Optimale Position der Zugschrauben bei Unterkiefermittenfraktur. b Kompression im Bruchspaltbereich durch Gleitlochprinzip am schraubenkopfnahen Fragment. c Kompressionskräfte an Vestibulär- und Lingualfläche bei Zugschraubenosteosynthese. d Kompression nach Applikation von zwei parallelen Zugschrauben bei schräger Unterkiefermittenfraktur.
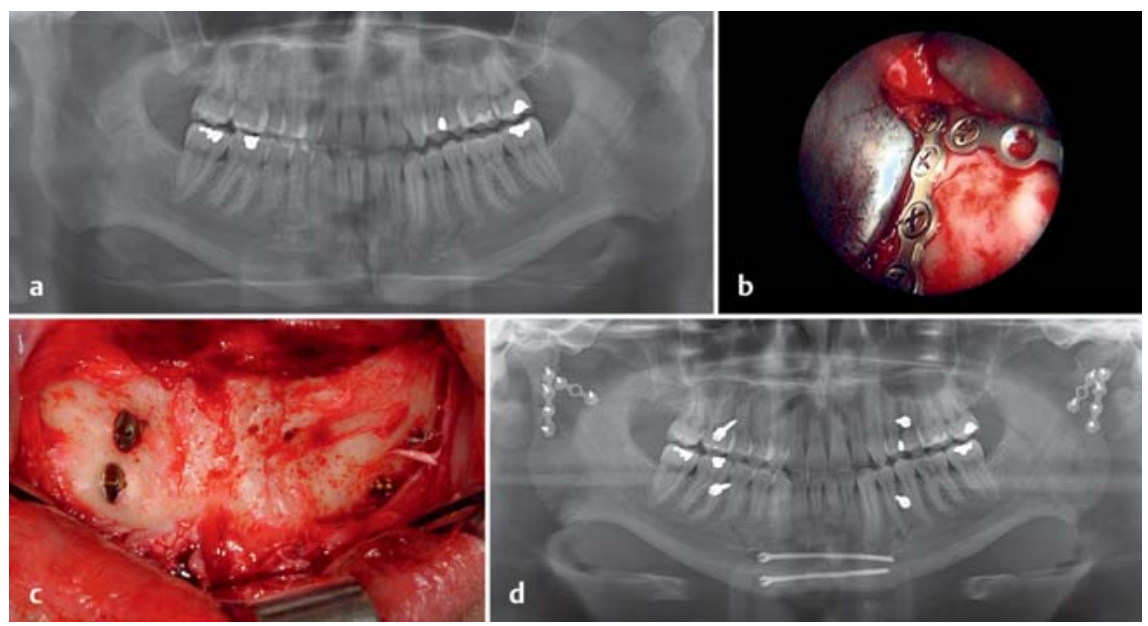

Abb. 14a bis d a Präoperative PSA einer „Open book“-Fraktur (rechtsseitig nach medial dislozierte Kiefergelenkhals-, linksseitig stark dislozierte Kiefergelenkhals- und dislozierte Unterkiefermedianfraktur). b Intraoperativer Situs mit 30-Optik nach Reposition und Plattenosteosynthese des Kiefergelenkfortsatzes rechts mit 2 Miniplatten bei endoskopischer Stellungskontrolle bei transoralem Zugang. c Intraoperativer Situs nach Reposition und Osteosynthese des Unterkieferkörpers mit 2 parallelen Zugschrauben. d Postoperative PSA nach Reposition und Osteosynthese der Kiefergelenkfortsätze beidseits mit jeweils 2 Miniplatten und der Unterkiefermitte mit 2 Zugschrauben.
Nach Darstellung aller Frakturzonen bringt es Vorteile, die Reposition und Fixation in der Reihenfolge Kiefergelenkfortsatzfrakturen zuerst, Kinnmitte zuletzt vorzunehmen.

Denn ein exakter Schluss der Frakturflächen in der Symphysen-/Parasymphysenregion ohne linguale Diastase lässt sich mit 2 instandgesetzten Hemimandibeln leichter vollziehen, da die Einstellung der prätraumatischen Okklusion sicherer wird, Rotationsfehler der Korpusfragmente vermieden werden und sich der Mandibularbogen mithilfe von Repositionsklemmen gut in Kongruenz bringen und halten lässt, bis eine stabile Osteosynthese durchgeführt ist.

\section{Reposition der Kiefergelenkfortsätze/ -walze}

Die Reposition der Fragmente erfolgt unter Distraktion am Kieferwinkel. Um eine anatomisch korrekte Position zu erreichen, ist eine Manipulation am proximalen Fragment mit geraden und/oder 

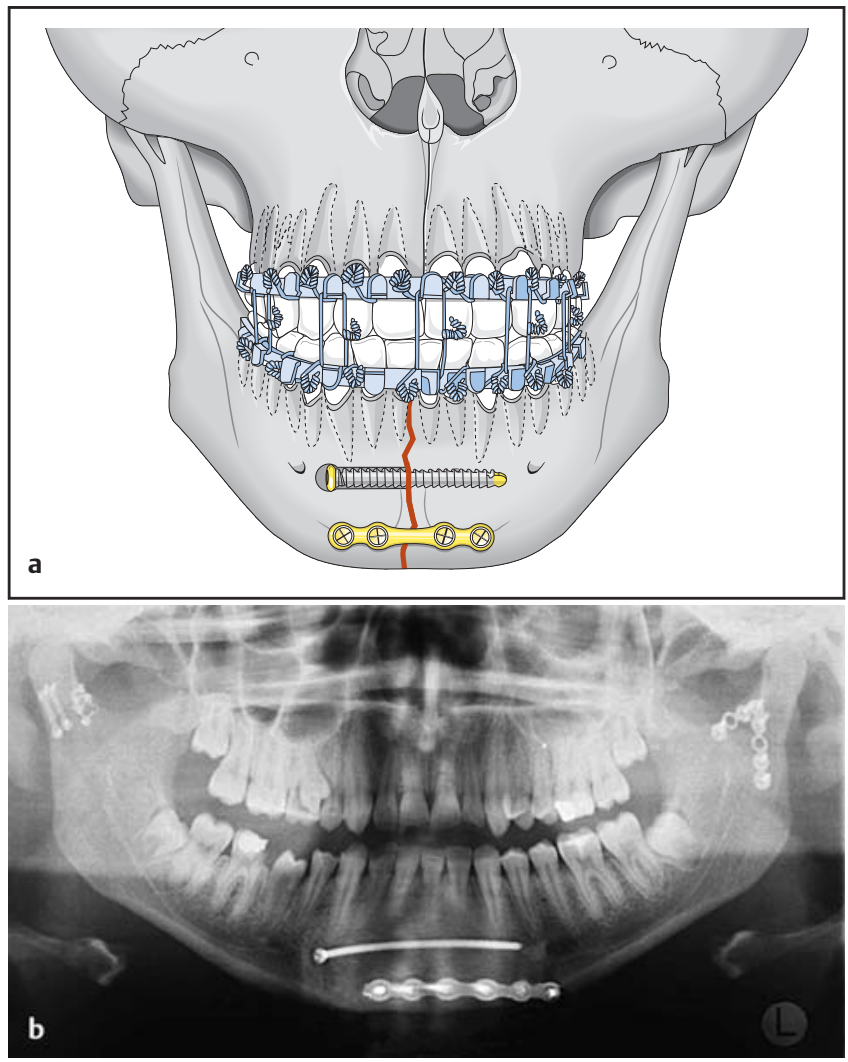

Abb. 15a und $\mathbf{b}$ a Kombination von Osteosyntheseplatte am Unterkieferunterrand und Zugschraube. b Postoperatives PSA nach Reposition mit Fixation der Kiefergelenkfortsätze beidseits durch Plattenosteosynthese und der Unterkiefermitte durch Kombination aus einer Osteosyntheseplatte und einer Zugschraube.

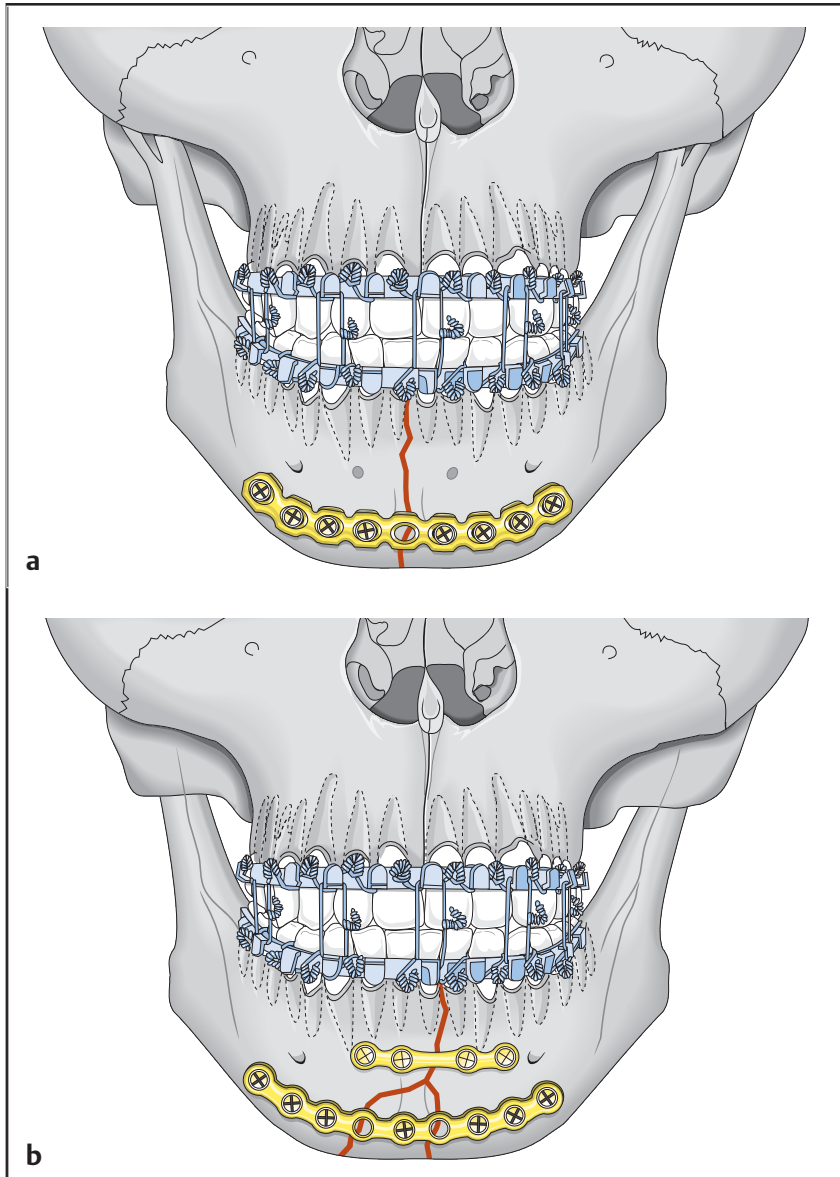

Abb. 16a und b a Plattenosteosynthese mit einer rigiden Osteosyntheseplatte am Unterkieferrand. b Plattenosteosynthese mit 2 Osteosyntheseplatten am Unterkieferrand und unterhalb der Zahnwurzeln. abgewinkelten Raspatorien solange erforderlich, bis eine achsgerechte Fragmentstellung erreicht ist. Vor der Osteosynthese ist die achsgerechte Stellung der Fragmente am Hinterrand des aufsteigenden Unterkieferasts $\mathrm{zu}$ kontrollieren. Die Reposition im Bereich der Gelenkwalze bedarf spezieller diamantierter Raspatorien und erfolgt in aller Regel unter vollständiger Relaxation des Patienten, um den die Reposition behindernden Muskelzug (M. pterygoideus lateralis) am proximalen Fragment zu reduzieren. Unzureichende nicht achsgerechte Repositionen der Kiefergelenkfortsätze führen aufgrund der vertikalen Verkürzung im Bereich der aufsteigenden Unterkieferäste zu einer Reduktion der hinteren Gesichtshöhe mit der Folge eines frontal offenen Bisses.

\section{Reposition Unterkieferhälften}

Die Reposition der Unterkieferhälften erfolgt manuell und unter zusätzlicher Verwendung einer Repositionszange, um Kompression auf den Bruchspalt aus- zuüben (Abb. 9). Es besteht aber die Gefahr, dass der Bruchspalt wegen gutem Schluss auf der Vestibulärseite geschlossen erscheint, obwohl auf der Lingualseite eine Diastase vorhanden ist. Klinisch bleibt diese Ausweichbewegung zunächst unbemerkt, da sich die damit einhergehende Disklusion auf die Innenseite der Okklusalflächen im Seitenzahngebiet beschränkt und sich einem Einblick entzieht. Die visuelle Kontrolle der Lingualseite ist über den üblicherweise gewählten transoralen vestibulären Zugang kaum möglich und eine palpatorische taktile Kontrolle mit dem Haken auf der Lingualseite gibt nur einen ungefähren Anhalt.

Dem Risiko einer Diastasenbildung auf der Lingualseite mit der Folge eines zu breiten Mandibularbogens kann durch das Einsetzen einer transoral eingebrachten großen Repositionszange (Abb. 10) im Kieferwinkelbereich entgegengewirkt werden. Durch einen kräftigen Druck im Bereich der Kieferwinkel bei gleichzeitigem ventralem Zug in der Symphyseal- region lässt sich die Gefahr einer lingualen Diastase nach Reposition ebenso reduzieren [17].

\section{Osteosynthese der Kiefergelenkfortsätze/-walzen}

Zur Fixation der Kiefergelenkfortsatzbasis und des Kiefergelenkhalses hat sich die Miniplattenosteosynthese durchgesetzt. Entsprechend der Untersuchung von Tillmann und Mitarbeiter [31] über die Druck- und Zugbelastungszonen am Gelenkfortsatz- und Ramusbereich mit der dicksten Kortikalis am lateralen Kiefergelenkfortsatz sollten 2 Miniplatten (vormals Stellplatte) in kaudaler Divergenzstellung angebracht werden, von denen eine am Hinterrand und die andere am Vorderrand des Kiefergelenkfortsatzes fixiert werden sollte (Abb. 11, 14 und 18). Um eine ausreichende Stabilität zu erreichen, sind mindestens 2 Schrauben pro Fragment zu inserieren. Alternativ kann eine einzelne rigidere Osteosyntheseplatte (vormals Kompressionsplatte) am Hinterrand des Kiefergelenkfort- 

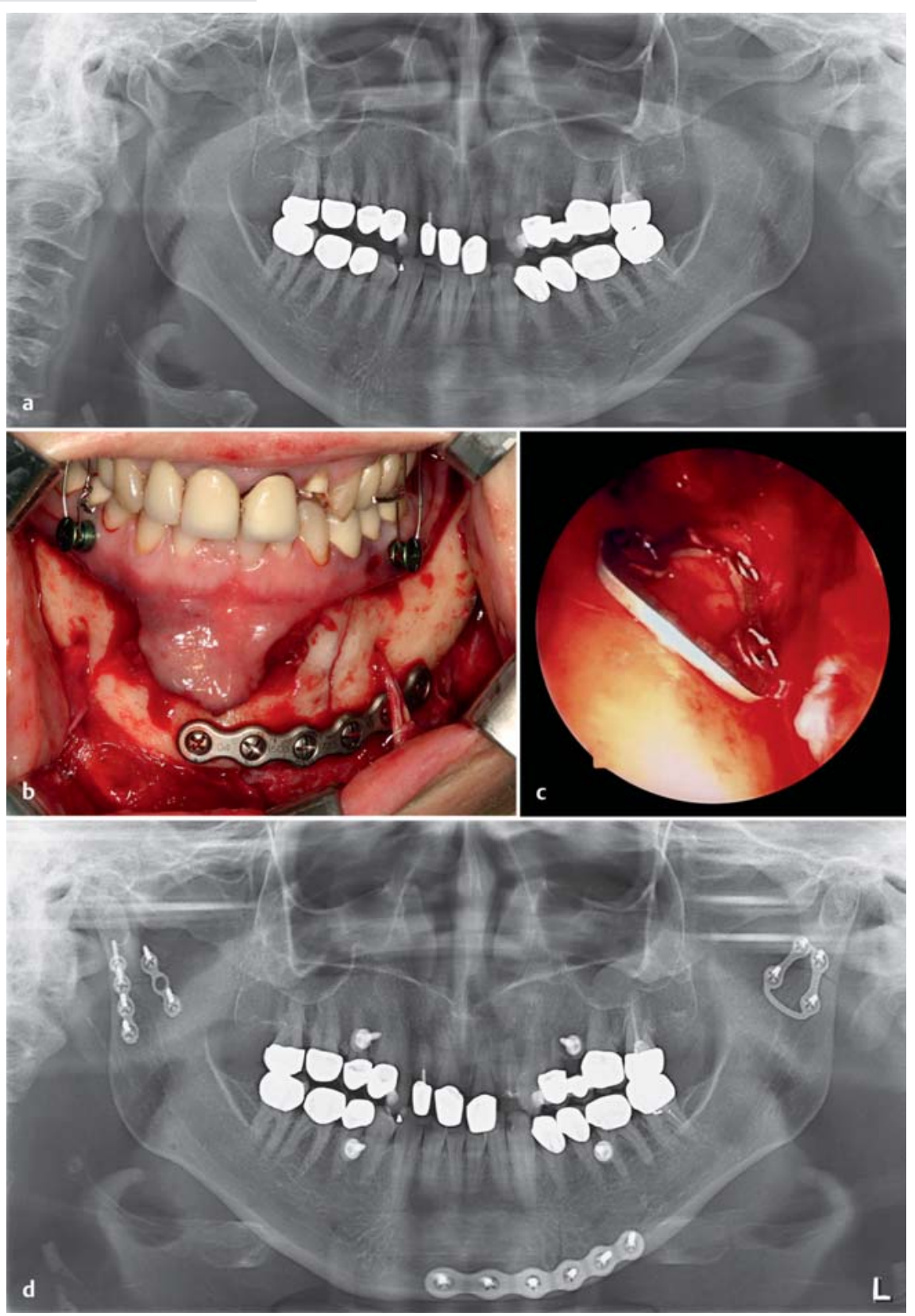

Abb. 17 a bis d a Präoperative PSA einer „Open book“-Fraktur (beidseitige dislozierte Kiefergelenkfortsatzbasisfraktur und dislozierte Unterkieferparamedianfraktur linksseitig). b Intraoperatives Situs nach Reposition und Osteosynthese mit einer rigiden Platte am Unterkieferrand. c Intraoperatives Bild mit $30^{\circ}$-Optik nach Reposition und Osteosynthese des Kiefergelenkfortsatzes links mit einer Subcondylar Trapezoid Plate unter endoskopischer Stellungskontrolle bei transoralem Zugang. d Postoperative PSA nach Reposition und Plattenosteosynthese im Bereich der Kiefergelenkfortsätze beidseits und Plattenosteosynthese am Unterkieferrand paramedian links.

satzes angebracht werden [32], wenn die Konfiguration eines schmalen Gelenkhalses die Positionierung einer weiteren 2. Platte nicht zulässt. Eine monokortikale Fixationstechnik der Osteosyntheseplatten ist dabei ausreichend. Mittlerweile stehen auch verschiedene spezielle für den Kiefergelenkfortsatz entwickelte Osteosyntheseplatten (sogenannte 3-DPlatten) von diversen Herstellern zur Verfügung (Abb.17 und 19), die sich bei einer Mikrostellplatte (Abb. 20). Dabei ist darauf zu achten, dass die Schrauben und Platten außerhalb der Gelenkfläche liegen.

\section{Osteosynthese der Unterkieferhälften}

Im Gegensatz zum Kiefergelenkfortsatz benötigt die Fixation der Unterkieferhälften aufgrund der Biomechanik eine stabilere und rigidere Fixation. Diese kann entweder mittels rigider Osteosyntheseplatten oder mittels Zugschrauben durchgeführt werden. Die optimale Position des Osteosynthesematerials zur Fixation der Unterkieferhälften ist in Abb. 12 dargestellt und entspricht dem Verlauf der Trajektorien am Unterkiefer.

\section{Zugschraubenosteosynthese}

Die Zugschraubenosteosynthese zur Fixation der Unterkieferhälften ermöglicht eine Kompressionsosteosynthese und bei anatomisch korrekter Reposition eine hohe Funktionsstabilität [11]. Dabei ist die Insertion von mindestens 2 Zugschrauben erforderlich (Abb.13a und 14), um Torsionskräften entgegenzuwirken. Die Kompression am Bruchspalt entsteht durch das Gleitlochprinzip im äußeren (schraubenkopfnahen) Fragment bei gleichzeitigem Fassen der Schraube im schraubenkopffernen Fragment (Abb. 13b). Dabei treten Kompressionskräfte sowohl an der Vestibulärwie an der Lingualfläche (Abb. 13b und c) des Bruchspalts auf, durch die bei exakter Reposition eine linguale Diastase sicher vermieden werden kann. Um ein Gleiten der Fragmente bei der Zugschraubenosteosynthese $\mathrm{zu}$ vermeiden, sollten die Zugschrauben möglichst senkrecht zum Bruchspalt inseriert werden (Abb. 13c und d). Zur Vermeidung einer Torsion kann statt einer 2. Zugschraube eine Osteosyntheseplatte am Unterkieferrand appliziert werden (Abb. 15). In ihrer retrospektiven Analyse konnten Tiwana und Mitarbeiter zeigen, dass eine Zugschraube im Bereich des Unterkieferrands eine vergleichbare Stabilität hat wie eine rigide Osteosyntheseplatte [35].

schmalem Kiefergelenkhals ohne Platz für eine weitere 2. Platte bewährt haben. Die Zugschraubenosteosynthese im Kiefergelenkfortsatzbereich mit ihren Modifikationen $[33,34]$ ist heutzutage als historisch anzusehen.

Die Fixation der Gelenkwalze erfolgt über 2-3 Kleinfragmentschrauben von lateral nach vorheriger arbiträrer Stellungsfixation der Gelenkfragmente mit

\section{Plattenosteosynthese}

Die günstigste Position der Osteosyntheseplatten am anterioren Unterkieferkörper wurde von Champy und Mitarbeiter für die Miniplattenosteosynthese festgelegt [36]. Entsprechend dieser Kraftlinien sollten am interforaminären Unterkieferkörper 2 Osteosyntheseplatten 


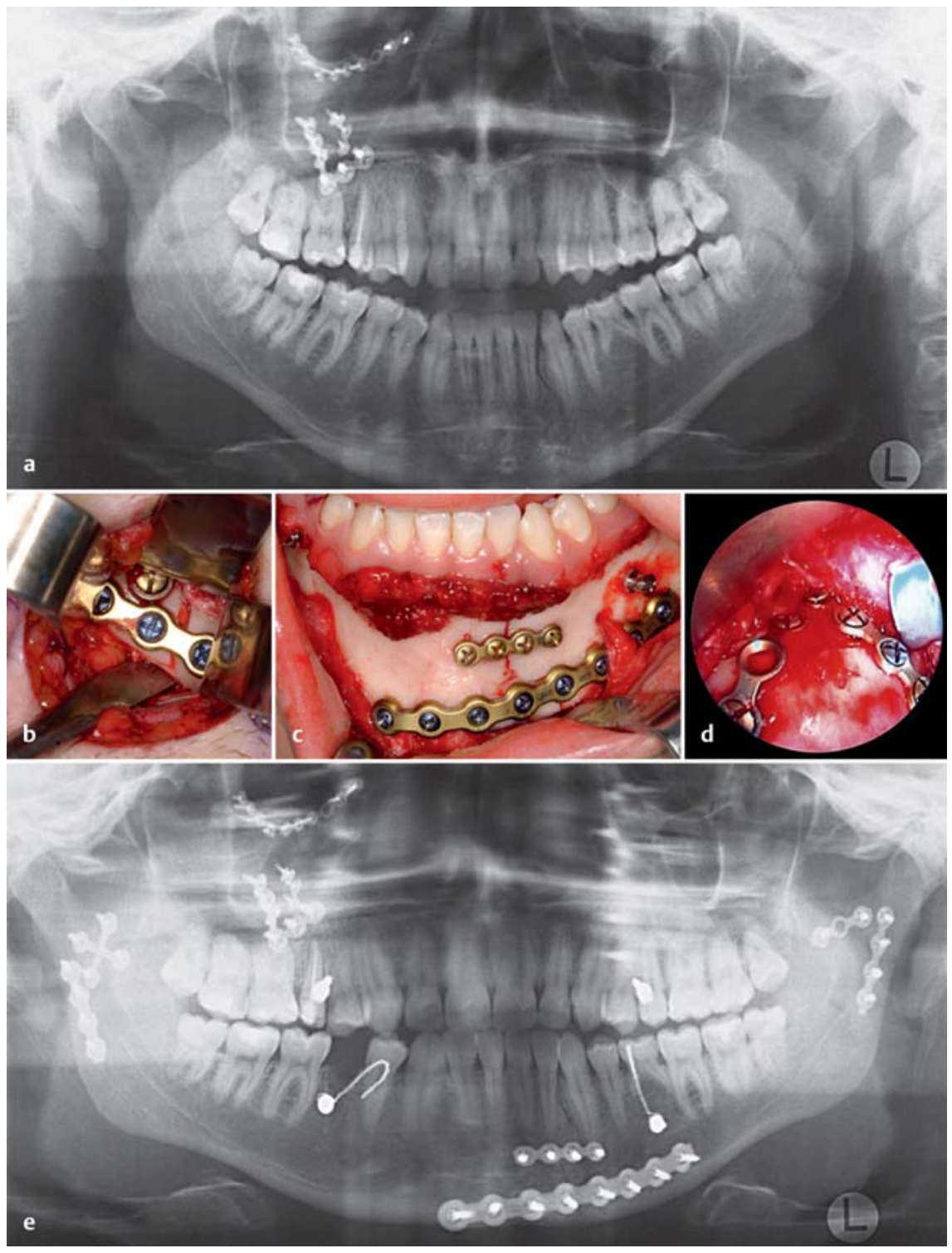

Abb. 18 a bis e a Präoperative PSA einer „Open book“-Fraktur (beidseitige dislozierte Kiefergelenkfortsatzbasisfraktur und dislozierte Unterkieferparamedianfraktur linksseitig) bei Z. n. Reposition und Plattenosteosynthese des Jochbeins rechts bei Jochbeinfraktur. $\mathbf{b}$ Intraoperatives Bild nach Reposition und Plattenosteosynthese des Kiefergelenkfortsatzes rechts mit 2 Miniplatten über transparotidealen Zugang. c Intraoperatives Bild nach Reposition und Osteosynthese der Unterkieferkörpers paramedian links mit 2 Platten. d Intraoperatives Bild mit 30-Optik nach Reposition und Plattenosteosynthese des Kiefergelenkfortsatzes links mit 2 Miniplatten bei endoskopischer Stellungskontrolle über transoralen Zugang. e Postoperative PSA nach Reposition und Fixation der Kiefergelenkfortsätze beidseits mit jeweils 2 Miniplatten und der Unterkiefermitte paramedian links mit 2 Osteosyntheseplatten.

verwendet werden, wobei eine am Unterkieferrand und die andere unterhalb der Zahnwurzeln der Unterkieferfrontzähne angebracht werden sollte. Um eine ausreichende Stabilität zu erreichen, sollten dabei jeweils mindestens 2 Schrauben auf jeder Fragmentseite in die Osteosyntheseplatte eingebracht werden. Ein Abstand von $5 \mathrm{~mm}$ zwischen den Platten hat sich als Optimum erwiesen, um Torsionsspannungen aufzunehmen. Aufgrund der besonderen Biomechanik
2 Osteosteosyntheseplatten (Abb. 16b, 18 und 19), von denen die rigidere am Unterkieferrand und die 2. unterhalb der Zahnwurzel positioniert wird. Ellis stellte in einer kürzlich publizierten Studie fest, dass die Komplikationsrate bei Verwendung einer rigideren Osteosyntheseplatte am Unterkieferrand geringer ist als bei 2 Platten [19].

\section{Resumée}

In der Argumentation gegen operative Behandlungsstrategien von UnterkieferDreifach- mit Beteiligung beider Gelenkfortsätze und der Unterkiefermittenfrakturen werden im Wesentlichen das Gefährdungspotenzial für den N. facialis bei den Zugängen zu den Kiefergelenkfortsätzen und sichtbare Narben im Gesichtsbereich angeführt, oftmals verknüpft mit der Behauptung, mit einer gezielten Physiotherapie gleich gute funktionelle Ergebnisse erreichen $\mathrm{zu}$ können.

Dennoch wird die Therapie nur noch in seltenen Ausnahmefällen in einer geschlossenen Therapie (Kieferbruchschienen zur MMF und Physiotherapie) bestehen. Auch ohne Dislokation der 4 Fragmente wird im Allgemeinen die Symphysenregion osteosynthetisch versorgt, um den raschen Beginn einer Physiotherapie zu gewährleisten. Wenn nicht mit einer sekundären Dislokation der Kiefergelenkfortsätze gerechnet werden muss, erscheint dies durchaus akzeptabel.

Bei den hier als „Open book“ titulierten stark dislozierten Unterkiefer-Dreifachfrakturen kommen konservative - allein auf eine Funktionswiederherstellung ausgerichtete - Therapiekonzepte prinzipiell aber nicht infrage, weil die Adaptationsleistung vorhersehbar nicht ausreichen wird, um die massiven Fehlstellungen vollständig zu kompensieren. Eine operative Versorgung der Symphysen-/Parasymphysenregion in Kombination mit nur 1 der beiden Kiefergelenkfortsatzfrakturen führt ebenfalls nicht zu regulären anatomischen Verhältnissen des Mandibularbogens und wird in funktioneller Hinsicht zwangsläufig kompromissbehaftet bleiben.

Nur eine aggressive operative Behandlungsstrategie mit Reposition und Fixation aller 3 Frakturen erscheint prognostisch aussichtsreich, denn das allein vermag die optimalen morphologischen Voraussetzungen für eine funktionelle Rehabilitation zu liefern. An vielen Klini- 


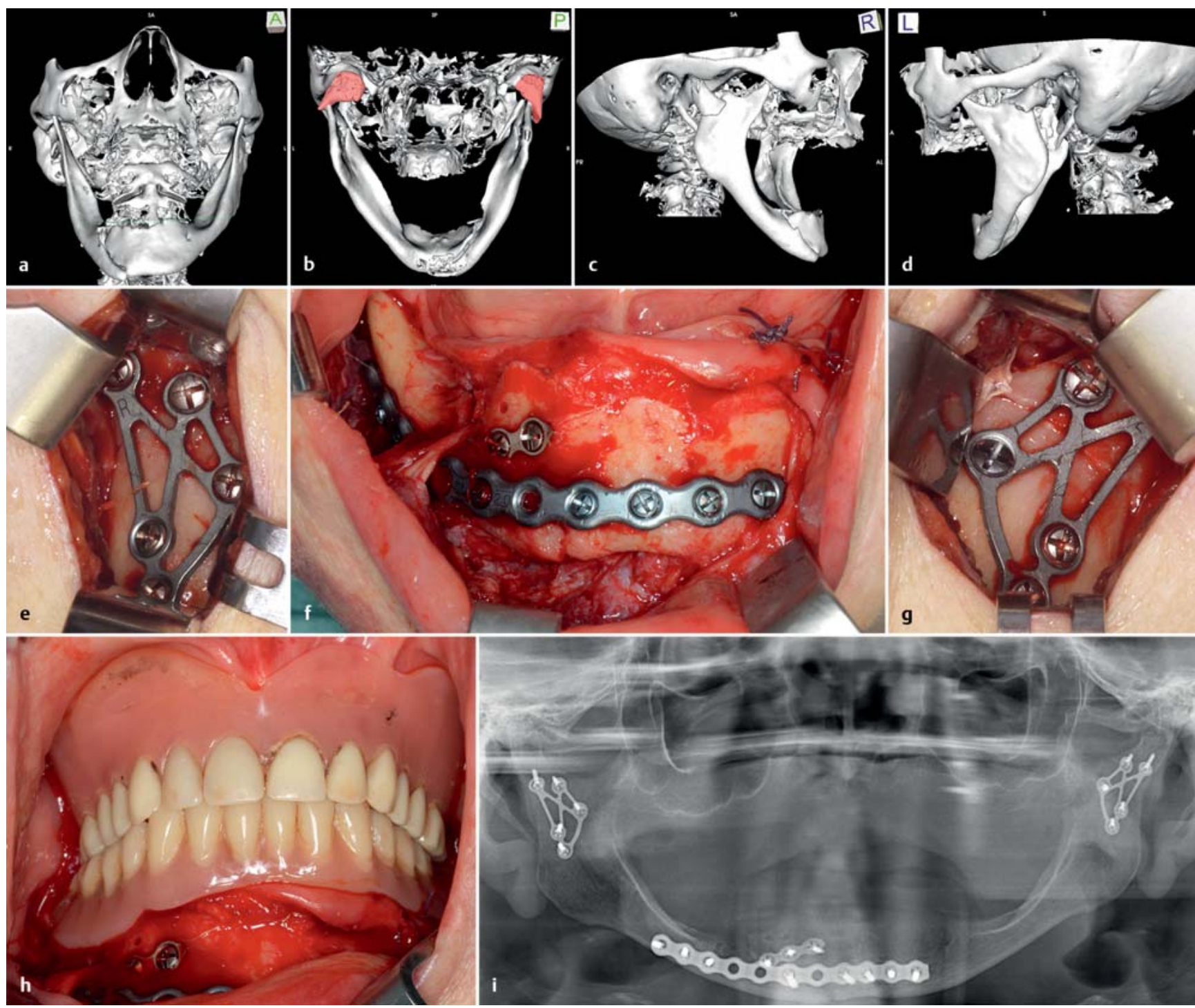

Abb. 19a bis i a Präoperatives 3-D-CT von frontal: Aufsprengung des Unterkieferkörpers paramedian rechts mit Aufweitung des Mandibularbogens. b Präoperatives 3-D-CT von dorsal: rechts laterale Dislokation mit Verkürzung des Gelenkfortsatzes, links Medialkippung des Gelenkfortsatzes mit Luxation aus der Pfanne. c Präoperatives 3-D-CT von rechts: laterale Dislokation mit Verkürzung des Gelenkfortsatzes rechts. d Präoperatives 3-D-CT von links: Medialkippung des Gelenkfortsatzes links mit Luxation aus der Pfanne. e Intraoperativer Situs nach Reposition und Plattenosteosynthese des Kiefergelenkfortsatzes rechts (Subcondylar Strut Plate) über transparotidealen Zugang. $\mathbf{f}$ Intraoperativer Situs nach Reposition und Plattenosteosynthese des Unterkieferkörpers paramedian rechts. g Intraoperativer Situs nach Reposition und Plattenosteosynthese des Kiefergelenkfortsatzes links (Subcondylar strut plate) über transparotidealen Zugang. $\mathbf{h}$ Intraoperativer Situs mit Okklusion der eingebrachten Ober- und Unterkieferprothesen nach Reposition und Plattenosteosynthese der „Open book“-Fraktur. i Postoperative PSA nach Reposition und Osteosynthese der Kiefergelenkfortsätze beidseits mit Subcondylar Strut Plate und des Unterkieferkörpers paramedian rechts mit 2 Osteosyntheseplatten.

ken haben sich die retromandibulären Zugangswege und dabei v.a. die transparotideale Dissektion sowie bei Vorhandensein eines Spezialinstrumentariums und hinreichender Erfahrung des OP-Teams der transorale Zugang zur Kiefergelenkfortsatzbasis und zum Kiefergelenkhals bewährt.

Hohe Gelenkhals- bzw. Gelenkwalzenabbruch und sagittal verlaufende Walzenfrakturen mit Höhenminderung des Ramus ascendens mandibulae sind nur über präaurikuläre oder auch über retroaurikulär transmeatale Zugänge darstell- bar, wobei diese Zugänge auch einen intraartikulären Einblick ggf. mit Reposition des Discus articularis gestatten [11, 37].

Nach Darstellung aller Frakturzonen bringt es Vorteile, die Reposition und Fixation in der Reihenfolge Kiefergelenkfortsatzfrakturen zuerst, Kinnmitte zuletzt vorzunehmen. Denn ein exakter Schluss der Frakturflächen in der Symphysen-/Parasymphysenregion ohne linguale Diastase lässt sich mit 2 instandgesetzten Hemimandibeln leichter vollziehen, da die Einstellung der prä- traumatischen Okklusion sicherer wird, Rotationsfehler der Korpusfragmente vermieden werden und sich der Mandibularbogen mithilfe von Repositionsklemmen gut in Kongruenz bringen und halten lässt, bis eine stabile Osteosynthese durchgeführt ist.

Seitdem die operative Versorgung von Kiefergelenkfortsatzfrakturen an Popularität gewonnen hat, konnten mehrere Studien zeigen, dass die Raten an peripheren Fazialisparesen [11,38-40] gering sind und es sich immer um kurzfristig reversible Nervenläsionen gehandelt 


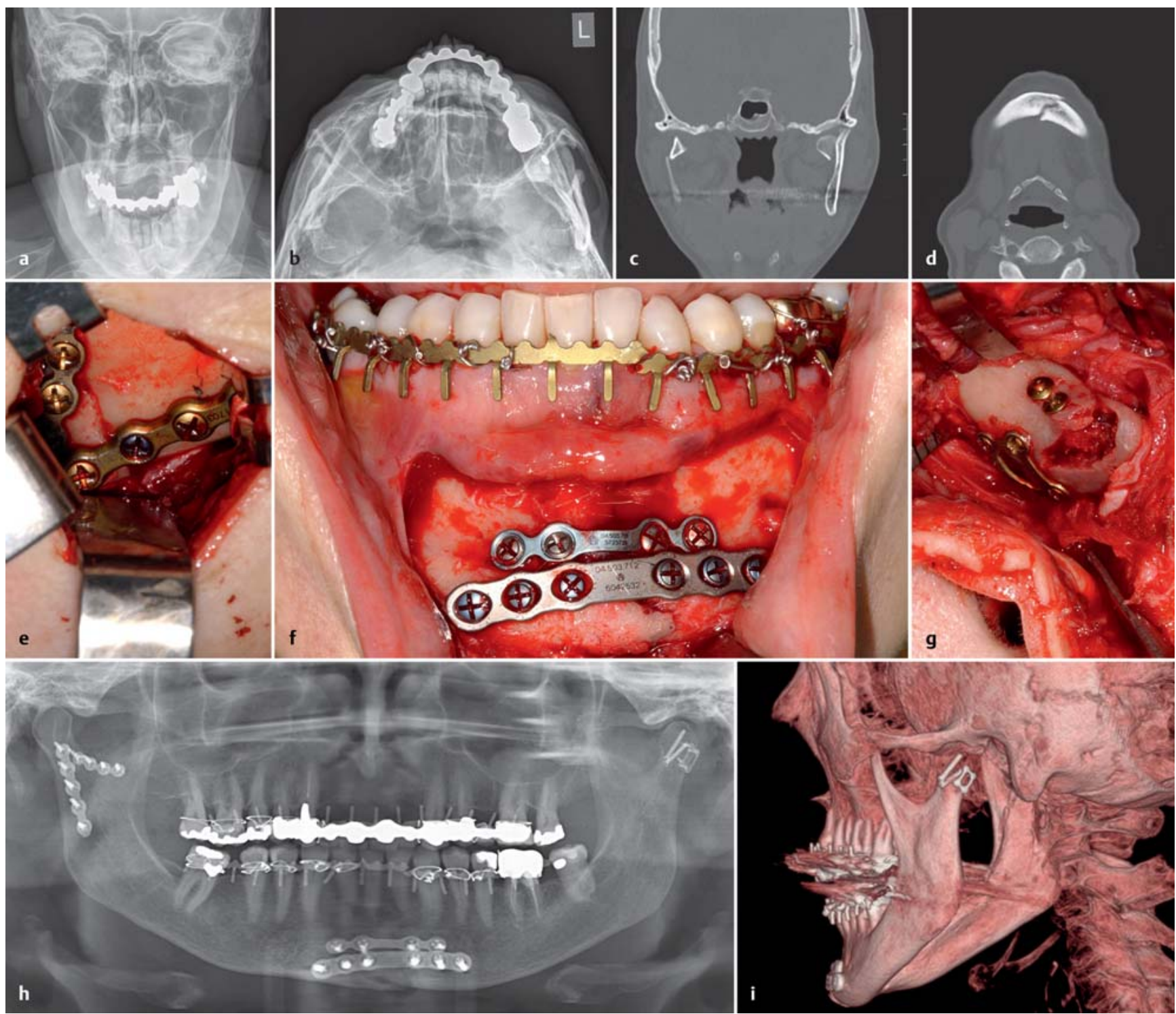

Abb. 20 a bis i a Präoperative Röntgenaufnahme nach Clementschitsch bei „Open book“-Fraktur, die Dislokation der Gelenkwalze lässt sich kaum erkennen. b Präoperative Röntgenaufnahme nach Runström mit Dislokation der Gelenkwalze links und des Kiefergelenkfortsatzes rechts. c Präoperatives CT koronal: medial dislozierte Kiefergelenkfortsatzfraktur rechts und Walzenfraktur links mit Höhenverlust. d Präoperatives CT axial: Dislokation des Unterkieferkörpers paramedian links. e Plattenosteosynthese des Kiefergelenkfortsatzes rechts über transparotidealen Zugang. f Plattenosteosynthese des Unterkieferkörpers paramedian links. g Osteosynthese der Gelenkwalzenfraktur (Stellplatte auf der Dorsalfläche in Kombination mit Schrauben von lateral unten) über einen aurikulär transtragalen Zugang. $\mathbf{h}$ Postoperative PSA nach Reposition und Osteosynthese aller Frakturen. i Postoperatives 3-D-CT nach Reposition und Osteosynthese der Gelenkwalzenfraktur (Stellplatte auf der Dorsalfläche in Kombination mit Schrauben von lateral unten).

hat. Bei Routineanwendung und steigenden Lernkurven dürfte sich die Häufigkeit der Fazialisparesen weiter senken lassen. Durch Auswahl und Modifikation der OP-Zugangswege lassen sich Narben im Gesichtsbereich darüber hinaus weitgehend kaschieren, sodass die althergebrachten Ressentiments gegen ein umfassendes operatives Vorgehen bei „Open book“-Frakturen des Unterkiefers entkräftet werden können. Führt man sich letztendlich die Schwierigkeiten und Risiken von Sekundäreingriffen zur Korrektur in Fehlstellung verheilter „Open book“-Frakturen ohne oder nach lediglich unilateraler operativer Versorgung der Kiefergelenkfortsatzfrakturen vor Augen, so ist es durchaus gerechtfertigt, die ORIF aller 3 Frakturen bei der „Open book“-Konstellation als absolute Indikation zu betrachten.

\section{Literatur}

${ }^{1}$ Huelke DF. Mechanics in the production of mandibular fractures: a study with the "stresscoat" technique. I. Symphyseal impacts. J Dent Res 1964; 43: 437-446

2 Huelke DF, Burdi AR, Eymen C. Mandibular fractures as related to site of trauma and state of dentition. J Dent Res 1961; 40: 1262-1266
${ }^{3}$ Huelke DF, Burdi AR, Eymen CE. Association between mandibular fractures and site of trauma, dentition and age. J Oral Surg Anesth Hosp Dent 1962; 20: 478-81

${ }^{4}$ Huelke DF, Harger JH. Maxillofacial injuries: their nature and mechanisms of production. J Oral Surg 1969; 27: 451-460

5 Huelke DF, Patrick LM. Mechanics in the production of mandibular fractures: straingauge measurements of impacts to the chin. J Dent Res 1964; 43: 437-446

6 Lindahl $L$. Condylar fractures of the mandible. I. Classification and relation to age, occlusion and concomitant injuries of teeth and teeth supporting structures and fractures of the mandibular body. Int J Oral Surg 1977; 6: $12-21$ 
7 Rowe NL, Killey HC. Fractures of the facial Skeleton. 2nd ed. Edinburgh: Churchill-Livingstone; 1968

8 Cope MR, Lawlor MG. An unusual mandibular dislocation. Br J Oral Maxillofac Surg 1985; 23: $112-117$

9 Petzel JR, Bulles G. Experimental studies of the fracture behaviour of the mandibular condylar process. J Maxillofac Surg 1981; 9: 211215

10 Loukota RA, Neff A, Rasse M. Nomenclature/ classification of fractures of the mandibular condylar head. Br J Oral Maxillofac Surg 2010; 48: 477-478

11 Rasse M. Neuere Entwicklungen der Therapie der Gelenkfortsatzbrüche der Mandibula. Mund Kiefer Gesichtschir 2000; 4: S69-S87

12 Neff A. Funktionsstabile Osteosynthese bei Frakturen der Kiefergelenkwalze: Ergebnisse experimenteller und klinischer Untersuchungen. Med. Habil. Schrift, Tech. Universität München 2002. Göttingen: Cuvillier: 2003

13 Neff A, Kolk A, Deppe H et al. Neue Aspekte zur Indikation der operativen Versorgung intraartikulärer und hoher Kiefergelenkluxationsfrakturen. Mund Kiefer Gesichtschir 1999; 3: S24

${ }^{14}$ Neff A, Kolk A, Hoch HH. Position und Beweglichkeit des Discus articularis nach operativer Versorgung intraartikulärer und hoher Kiefergelenkluxationsfrakturen. Mund Kiefer Gesichtschir 2000; 4: S111

15 Schneider M, Erasmus F, Gerlach KL et al. Open reduction and internal fixation versus closed treatment and mandibulomaxillary fixation of fractures of the mandibular condylar process: a randomized, prospective, multicenter study with special evaluation of fracture level. J Oral Maxillofac Surg 2008; 66: 25372544

16 Singh V, Bhagol A, Dhingra R. A comparative clinical evaluation of the outcome of patients treated for bilateral fracture of the mandibular condyles. J Craniomaxillofac Surg 2011 August [Epub ahead of print]

17 Ellis 3rd E, Tharanon W. Facial width problems associated with rigid fixation of mandibular fractures: case reports. J Oral Maxillofac Surg 1992; 50: 87-94

18 Gerbino G, Boffano P, Bosco GF. Symphyseal mandibular fractures associated with bicondylar fractures: a retrospective analysis. J Oral Maxillofac Surg 2009; 67: 1656-1660

19 Ellis 3rd E. Is lag screw fixation superior to plate fixation to treat fractures of the mandibular symphysis? J Oral Maxillofac Surg 2012; 70: 875-882

20 Scolozzi P, Richter M. Treatment of severe mandibular fractures using AO reconstruction plates. J Oral Maxillofac Surg 2003; 61: 458

21 Neff A, Kolk A, Meschke F et al. Kleinfragmentschrauben vs. Plattenosteosynthese bei Gelenkwalzenfrakturen - Vergleich funktioneller Ergebnisse mit MRT und Achsiographie. Mund Kiefer Gesichtschir 2005; 9: S80
${ }^{22}$ Eckelt U. Gelenkfortsatzfrakturen. Mund Kiefer Gesichtschir 2000; 4 (Suppl.1): S110S117

23 Ellis 3rd E, Zide MF. Retromandibular Approach. In: Ellis 3rd E, Zide MF, eds. Surgical Approaches to the facial Skeleton. Baltimore: Williams \& Wilkins; 1995: 139

24 Silverman $S$. A new operation for displaced fractures at the neck of the mandibular condyle. Dental Cosmos 1925; 67: 876-877

25 Fritzemeier CU, Bechthold $H$. Unterkiefergelenkfortsatzfrakturen mit alleinigem Zugang von intraoral. Mund Kiefer Gesichtschirurgie 1993; 17: 66-70

26 Schön R, Gutwald R, Schramm A et al. Endoscopy-assisted open treatment of condylar fractures of the mandible: extraoral vs. intraoral approach. Int J Oral Maxillofac Surg 2002; 31: 237-243

27 Schmelzeisen R, Cienfuegos-Monroy R, Schon $R$ et al. Patient benefit from endoscopically assisted fixation of condylar neck fractures - a randomized controlled trial. J Oral Maxillofac Surg 2009; 67: 147-158

28 Schön R, Fakler O, Metzger MC et al. Preliminary functional results of endoscope-assisted transoral treatment of displaced bilateral condylar mandible fractures. Int J Oral Maxillofac Surg 2008; 37: 111-116

${ }^{29}$ Hoffmann A, Mast G, Ehrenfeld M. Verwendung von IMF-Schrauben zur mandibulomaxillären Fixation. OP-Journal 2003; 19: 70-75

30 Arthur G, Berardo N. A simplified technique of maxillomandibular fixation. J Oral Maxillofac Surg 1989; 47: 1234

31 Tillmann B, Härle F, Schleicher A. Biomechanik des Unterkiefers. Dtsch Zahnärztl Z 1983; 38 : 285-293

32 Hammer B, Schier P, Prein J. Osteosynthesis of condylar neck fractures: a review of 30 patients. Br J Oral Maxillofac Surg 1997; 35: 288-291

33 Eckelt U. Zur funktionsstabilen Osteosynthese bei Unterkiefergelenkfortsatzfrakturen. Universität Dresden: Habilitationsschrift; 1984

${ }^{34}$ Krenkel C. Axial "anchor" screw (lag screw with biconcave washer) of "slanted-screw" plate for osteosynthesis of fractures of the mandibular condylar process. J Craniomaxillofac Surg 1992; 20: 348-353

35 Tiwana PS, Kushner GM, Alpert B. Lag screw fixation of anterior mandibular fractures: A retrospective analysis of intraoperative and postoperative complications. J Oral Maxillofac Surg 2007; 65: 1180

36 Champy M, Loddé JP, Schmitt R et al. Mandibular osteosynthesis by miniature screwed plates via a buccal approach. J Maxillofac Surg 1978; 6: 14-21

37 Umstadt HE, Ellers M, Müller $H H$ et al. Functional reconstruction of the TM joint in case of severely displaced fractures and fracture dislocation. J Caniomaxillofac Surg 2000; 28: 97-105
38 Vogt A, Roser M, Weingart D. Transparotidean approach to surgical management of condylar neck fractures. A prospective study [Article in German]. Mund Kiefer Gesichtschir 2005; 9: 246-250

39 Biglioli F, Coletti G. Transmasseter approach to conylar fractures by miniretromandibular access. J Oral Maxillofac Surg 2009; 67: 24182424

40 Klatt J. Heiland M, Blessman $M$ et al. Clinical indication for intraoperative 3D imaging during open reduction of fractures of the neck and head of the mandibular condyle. J Craniomaxillofac Surg 2011; 39: 244-248

\section{Dr. med. Dr. med. dent Sebastian Schiel \\ Oberarzt \\ Dr. med. Dr. med. dent. Wenko Smolka \\ Oberarzt \\ Prof. Dr. med. dent. \\ Gabriele Kaeppler}

Leitung Röntgenabteilung und

Fachzahnärztin für Oralchirurgie

Prof. Dr. med. Dr. med. dent. Carl Peter Cornelius

Oberarzt

Klinik und Poliklinik für Mund-, Kiefer- und Gesichtschirurgie Klinikum der Universität München Lindwurmstraße 2 a

80337 München

sebastian.schiel@

med.uni-muenchen.de

PD. Dr. med. Dr. med. dent. Christoph Leiggener Oberarzt

Klinik für Mund-, Kieferund Gesichtschirurgie Universitätsspital Basel Spitalstr. 1

4031 Basel

Schweiz 